Master Thesis

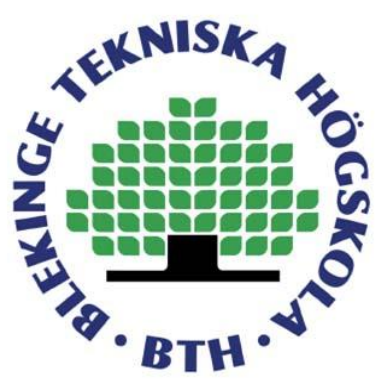

In Electrical Engineering with Emphasis on Telecommunications

\title{
Emergency based remote collateral tracking system using Google's Android mobile platform
}

$\begin{array}{lr}\text { Ashokkumar Ramalingam } & \text { 870504-9537 } \\ \text { Prabhu Dorairaj } & 851218-1531 \\ \text { Saranya Ramamoorthy } & 850319-8486\end{array}$

Under the Guidance of

Prof. Abbas Mohammed

Blekinge Institute of Technology

September 2011

Department of Electrical Engineering

School of Engineering

Blekinge Institute of technology

SE-371 79 Karlskrona

Sweden 


\begin{abstract}
Introduction of Smart phones redefined the usage of mobile phones in the communication world. Smart phones are equipped with various sophisticated features such as Wi-Fi, GPS navigation, high resolution camera, touch screen with broadband access which helps the mobile phone users to keep in touch with the modern world. Many of these features are primarily integrated with the mobile operating system which is out of reach to public, by which the users can't manipulate those features. Google came up with an innovative operation system termed as ANDROID, which is an open system architecture with customizable third party development and debugging environment which helps the user's to manipulate the features and to create their own customizable applications.

In this thesis, 'Emergency Based Remote Collateral Tracking System' application using Google's Android Mobile Platform is addressed. Emergency is divided into three categories: heart beat based emergency, security threats like personal safety and road accidents. This application is targeted to a person who is driving a vehicle. Heart rate monitoring device is integrated with our application to sense the heart beat of a person driving the vehicle and if there is any abnormalities in the heart beat, then our application performs a dual role. One in which, application uses a GPS to track the location information of the user and send those location information as a message via SMS, email and post it on Facebook wall Simultaneously, an emergency signal is sent to Arduino Microcontroller.

Road accidents are quite common, this application is also designed to detect the accident using the sensors in the Android Mobile. Security threat can occur anywhere, our application also answers for personal safety, when the user interacts with the application by pressing the button, then automatically the application generates the geographical information and sends that location information via SMS and email to a pre-stored emergency contact and the same information will be posted on user's Facebook wall. This application is written in JAVA programming language which runs on Eclipse Integrated Development Kit.
\end{abstract}

Key words: Android, Arduino microcontroller, Emergency, GPS, Heart rate device 


\section{Acknowledgement}

I, Prabhu Dorairaj very thankful to Prof. Abbas Mohammed for all his support and guidance on completing this thesis in stipulated time. 'A friend in need is a friend indeed', Special thanks to all my dear friends from A-Z who were there in all occasion. I would also like to thank BTH staff and the department for giving me the opportunity to join such a program. I express my gratitude to my fellow thesis partners Mr. Ashok and Mrs. Saranya for their timely support. Heart filled thanks to Mr.Mikeal Åsman and Selva kumar Ramachandran who introduced a term called ANDROID to me. Exceptional thanks to Swarna Gowri for her enthusiastic support during my Masters. Last but by no means least, I would like to thank my dad, mom, chittappa, chitti and my dear brothers, for whom this thesis is dedicated. For their continued support, encouragement and sacrifice throughout the years, I will be forever indebted to them for all that they have done.

I, Ashokkumar Ramalingam, am very happy to do thesis under Prof. Abbas Mohammed. His nice support and advices were really helpful to complete this thesis. I would like to thank Mr. Mikeal Åsman, all BTH peers and staffs. It's a good experience to study \& learn several things with modern Institute. Finally I would like thank God to complete this thesis successfully. Special thanks to my amma (Menakadevi), appa (Ramalingam), cool anna (Vanjinathan) \& akka (Subashini) for their moral and timely support to me. Further I would like thanks to Prabhu Dorairaj with his wonderful support, guidance and help throughout my Master degree. Also I am very happy to say thanks to Annette Björck, Mikael Malmberg for their wonderful help to stay in Sweden. Apart from this, I would like to thank my cousin Dr.Duraivelu , Saranya Ramamoorthy, Gunasekar, Swarna Gowri, Arunkumar Jayaraman, Swarnalatha and all my Sweden roommates.

I, Saranya Ramamoorthy would like to express my heartfelt gratitude to my Supervisor Prof. Abbas Mohammed for being very encouraging and supportive throughout which helped us to complete the thesis very smoothly. Special thanks to Mr.Gurudatt Velpula, International Coordinator for giving an opportunity and providing the exposure to study in BTH. I would like to thank Mr. Mikeal Asman for his valuable suggestions and guidance during the entire study period. I would also like to thank all the professors who have made this course very interesting for me to learn and explore new things. Most importantly I would like to thank my husband Ganesh Subramaniam who has encouraged me to join this course. I would like to extend my sincere thanks to my mother, brother and my in laws for supporting me in all times. It gives me immense pleasure to thank all my friends and roommates who has made this journey so memorable. Last but not least I would like to thank Prabhu Dorairaj and Ashok Ramamlingam for being such a wonderful Thesis partners who has been a great strength, which has helped in the successful completion of the Thesis. 


\section{Table of contents}

1. Introduction 8

1.1 Problem Statement

1.2 Thesis Scope 9

1.3 Thesis Outline 10

1.4 Historical development of mobile operating system

2. Literature review

2.1 What is Android? 14

2.2 Features incorporated with Android 14

2.3 Components of Android operating system _

2.4 Android Manifest _... 20

2.5 Arduino Microcontroller _. 23

2.6 Integration of Arduino with Android-Amarino _. 25

2.7 Heart Rate Monitoring Device

3. Research Methodology 27

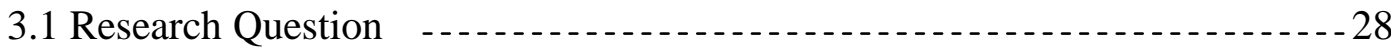

3.2 Expected Outcomes 28

3.3 Main Contribution 28

4. Problem Solution 29

4.1 Heart rate based 29

4.1.1Problem Statement _. 29

4.1.2Scenario (2.

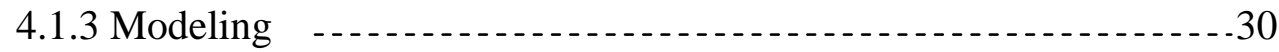

4.1.4 Implementation and Validation _. 31

4.1.4.1 Implementation of API with Heart Rate Device _....... 31

4.1.4.2 HR device communications Link _... 
4.2 Security Threats $-38$

4.2.1 Problem Statement _. 38

4.2.2 Scenario

4.2.3 Modeling - 38

4.2.4 Implementation and Validation

4.3 Accidents _. 42

4.3.1 Problem Statement _.

4.3.2 Scenario _ _ 42

4.3.3 Modeling -

4.3.4 Implementation and Validation

5. Results

6. Conclusions

7. References 


\section{List of Figures}

1. History of mobile operating system

2. Components of Android operating system

3. Project Folder Structure in Eclipse $\ldots 20$

4. Sample AndroidManifest.xml file

5. Arduino BT Microcontroller $\ldots . .24$

6. Zephyr heart rate monitoring device

7. Emergency Condition Scenarios _. 27

8. Modeling of heart rate based scenario

9. Communication between Zephyr HR device and Android Mobile Phone . . . . _ _ 31

10. HR device communication link _. 33

11. Zephyr HR device worn -

12. ETS Application

13. Entire Workflow of Pairing HR device to Android phone

14. Enter Details page 35

15. Abnormal heart rate scenario - workflow

16. Message sent via SMS, Mail and Facebook Wall _.

17. Modelling of Personal safety Scenario

18. Implementation of Personal Safety Scenario-

19. Workflow of Personal Safety Scenario _

20. Modelling of Accident Scenario

21. Movement of Mobile

22. Sensing the movement of mobile using Accelerometer

23. Working and Result of ETS Application 


\section{Table of Abbreviations}

\begin{tabular}{ll} 
Abbreviations & \multicolumn{1}{c}{ Description } \\
API & Application Interface Programming \\
BPM & Beats Per Minute \\
ETS & Emergency Tracking System \\
GPS & Global Positioning System \\
HR & Heart Rate \\
IDE & Integrated Development Kit \\
JVM & Java Virtual Machine \\
MMS & Multimedia Messaging Service \\
OHA & Open Handset Alliance \\
OS & Operating System \\
PDA & Personal Digital Assistants \\
RIM & Research In Motion \\
SD & Secure Digital \\
SDK & Software Development Kit \\
SMS & Short Message Service \\
UI & User Inerface
\end{tabular}




\section{Chapter 1}

\section{Introduction}

Instauration of mobile devices gave birth to lot of innovative technology, and exchanging information globally has become more prominent. Smart phones gave a new dimension to the usage of mobile phones for the users. Apart from basic functionality such as messaging, calling and cameras, smart phones laid a way to portray a personal computer. Not only the mobile phone looks newer, it's the operations system and the applications which are built to meet the various features of the hardware made difference.

The mobile phone has now become a major source of information device which can be seen almost in everyone's hand in the world. Mobile devices with computing process ability have been widely used to access network via mobile communication network. Different categories of application such as games, social networks, and health care are being developed to meet the user's requirements. Each mobile user is of unique kind, one wants to use the basic functionality of the smart phones, the other want to use the built in application, the most advanced user who wants to play with the hardware and to develop his own customizable application. To answer each kind of user, Google mustered up a groundbreaking product called as "ANDROID", which includes an open source operating system, middleware and a user-interface $[1,3]$. 


\subsection{Problem statement}

There are four major smart phone operating systems which rule the mobile world namely, Symbian, Windows, iPhoneOS and Android [1]. Out of them, Android is a new and yet dynamically developing mobile platform and almost all counterpart of application are being adopted to compact the system's requirement. GPS is one of the notorious application. The use of GPS on mobile devices in the recent generations of mobile communication is one of the ubiquitous applications that are widely developed and used. But using those GPS in case of emergency where there is no user interaction will be an arduous effort and hence ANDROID can answer for this, by developing an application which can solve the above mentioned problem.

\subsection{Thesis scope}

The main objective of this thesis is to explore an open source mobile operating system called as ANDROID, and its features such as GPS, Internet capability to serve person under critical situation by developing an intelligent application based on android operating system. Furthermore, a heart rate monitoring device is integrated with this application to ensure safety with regards to the person heartbeats.

Moreover, a new yet dynamically developing open source microcontroller platform called as ARDUINO is also presented here, which plays a major role in this project. Arudino microcontroller is integrated with the mobile via Bluetooth. Arduino board based micro controller programming is also performed to send an emergency signal when person is under critical situation.

This project is developed with JAVA programming language which is executed on Eclipse IDE and implemented on Android mobile platform. A typical Graphical User Interface is used to manage the scenario and make it eventually available to the public at large. 


\subsection{Thesis Outline}

In chapter 2, we will discuss the various technical aspects used in our thesis, such as Android mobile operating system, Arduino microcontroller, heart rate monitoring device and its diverse usage in our thesis.

In chapter 3, we deal with research methodology by developing our research question and creating the respective hypothesis. This chapter also describes about the main contribution of our thesis.

In chapter 4, we describe about the different cases of implementation and validation part, which starts from heart rate based, security and accidents.

In chapter 5, the result of different scenarios will be analyzed and discussed.

In Chapter 6, we conclude the work done in this thesis. 


\subsection{History of mobile operating system}

Operating system is the heart of mobile devices, which controls and interacts with the mobile hardware. Similar precept to an operating system such as Windows, Mac OS and Linux, that controls the desktop or laptop. Device which runs on operating system are smart phones, PDA's and tablet computers.

Everyone wants to do everything fast and on the go. When people where sitting back and diddling with the heavy computers for accessing the internet. An operating system called Palm OS was launched in year 1996 which brought a drastic change in the communication world [2]. With the introduction of Palm OS 2.0 in the year 1997, accessing and sending mail via mobile evolved. The time when Palm OS was standing alone in the Smart phone market in the year 2000, another giant bounced into the market, introducing Windows "Pocket PC 2000" which almost had most of the features of a computer.

Entertainment on the go was achievable with windows by launching "Pocket PC 2002" which incorporated MSN messenger and media player with enhanced user interface. Bluetooth an extraordinary invention for file transfer wirelessly. Bluetooth integration was successfully implemented in Windows Mobile 2003 and browsing was made more comfortable with the pocket internet explorer. When windows were acquiring the smart phones market, Palm OS Cobalt bounced back with wifi and Bluetooth connectivity in 2004.

In 2005, Google acquired the Android Inc and Blackberry's OS 4.1 was made available in the market. Windows interfaced the GPS management and office mobile in their "windows mobile 5". When everyone was going upwards in updating the version and integrating application in the smart phones. The release of "iPhone" in 2007 disrupted the mobile industry and gave a new era of smart phone operating system with user experience which relies on touch based user interaction.

In 2007, a trendsetting year when Google formed the OHA [4] with 79 other hardware, software and telecommunication companies to make entry in to the smart phone market by introducing a legendary open source operating system "ANDROID" resulted in 2008 with Android 1.0 which was available in the market. Android came up with a middleware which is responsible for hardware and communication between applications, and 
provides open source Android SDK application that allows embedded systems developers to use it to develop their own customizable Android platform applications. Some notable top applications such as Google map, E-mail, Instant messaging, Browser, GPS, Multimedia messaging are widely made available to the people in large only because of Android.

The enhancing grandness of smart phones has sparked off intense contenders amongst software giants such as Google, Microsoft, and Apple, as well as mobile industry leaders Nokia, RIM, and Palm to keep on updating their technology. In 2009, Samsung too joined the roads of smart phones when they released a new operating system called as BADA platform. Nevertheless hewlett packard Web OS was also introduced in the same year. But Google's Android was climbing so high in a year, they acquired the major share in the smart phone operating system by upgrading from Android 1.0, 1.1-1.6 till 2.1 (Éclair) and version 3.1 (Honeycomb) was released in 2011 [2]. 

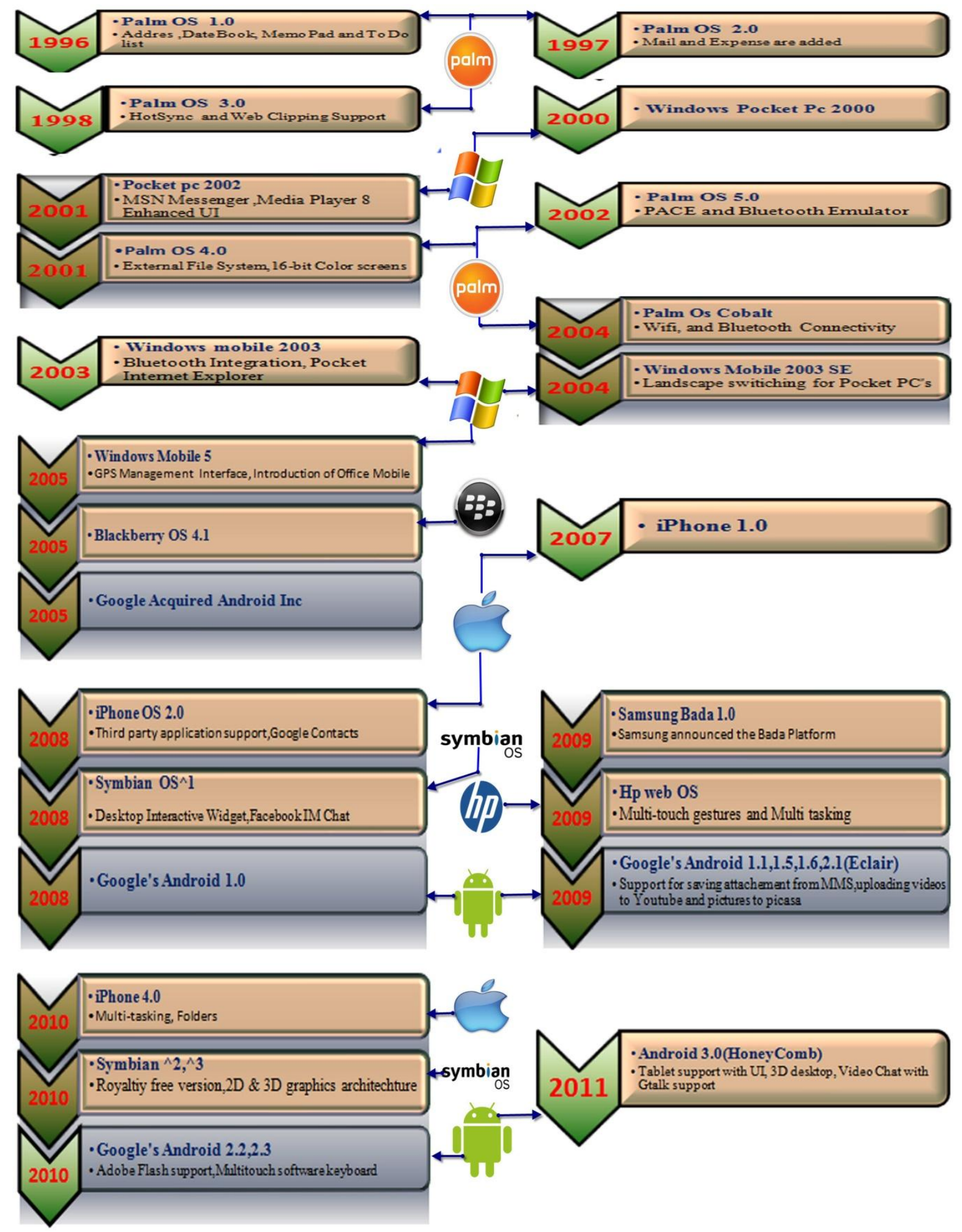

Figure 1 History of Mobile Operating System 


\section{Chapter 2}

\section{Literature review}

\subsection{What is Android?}

In 2005, Google acquired Android from Android Inc. which was found in year 2003 by Andy Rubin and they dealt with developing software for mobile devices. Later, OHA which comprises of 79 companies along with Google developed their new mobile platform for mobile devices. This alliance was formed so as to develop open technologies for mobile devices and make those applications easily available in the market. This new open source technology was named as Android [3, 4].

Android is an open source architecture which is used for developing applications for mobile devices. Android works on Linux Kernel. It has an operating system, middleware and key applications. Android announced its code under the license of free software/open source in the year 2008. Android comes up with an API for mobile devices. This Linux Kernel supports Java Virtual Machine which favours Java to be most suitable programming Language for development of the applications. Google provides a SDK to all developers which include libraries, debugger and a handset emulator in Eclipse IDE [5, 6]. The application which is developed in Android can be tested using this emulator which works similar to a mobile phone.

\subsection{Features incorporated with Android}

Android supports various built in and developed features which divides it into categories as hardware and software features. Hardware lineaments are the built in features of the phone which is been used by android to greater extent. Software lineaments are the one which is been developed by the user as per their requirements. To mention a few, the hardware lineaments are Camera, GPS, Touch screen, Accelerometer, SD Card, whereas the software lineaments are Internet, Audio and Video, Messaging, Contacts and so on [7,8] . Few of these features show cases the significance of Android and these are discussed in detail below. 


\subsubsection{GPS}

GPS is a space based satellite system which will provide the location and time anywhere on earth in any weather when the line of sight of the GPS satellites is unhampered [6]. This facility is invoked using a GPS Radio of the phone along with the Android operating system which helps to detect the location of the user. As and when the user moves from one place to another, the change of location is also traced and displayed. GPS is implemented using the SDK provided by Google along with support of Google maps. This SDK will detect the user's location by invoking the class LocationManager [9].

\subsubsection{Accelerometer}

Another feature of the android is Accelerometer. Accelerometer, an inbuilt sensor which is available in mobile phone and it is also supported by android. Accelerometer measures the acceleration of the object. It detects the acceleration experienced by the fall of the object or any slight movement in the object. In terms of Physics, it can be said that accelerometer measures weight per unit of the quantity being measured. This sensor [10] would help to detect any accidents occurring with the help of the movement of the object in which the sensor is fixed and in our context, it is a mobile phone. This acceleration is analysed by invoking the class SensorManager [9] in the SDK provided by Google.

\subsubsection{Messaging}

Messaging or even called as instant messaging is a form of communication where a text based information is been communicated among two persons. A mobile phone enabled with Android supports SMS and MMS form of messaging [7]. The messaging service is invoked by the class Message [9] using the Google's SDK. Using this class, the message is sent to the destination number which is pre-stored and can be fetched from the phone's contact list. 


\subsubsection{Internet}

Internet has become the backbone for all innovation, knowledge and information. In today's world nothing is possible without internet. Every single info is available. Android has also handled the internet facility in better way. Starting from the installation of JVM, internet is been widely used. Android has taken the help of internet in various ways for its applications [11]. To site few examples, like location of the person with the Google Maps [5], location of nearest movie halls, hospitals or any community buildings. It is also used to find the timing of the public transport system, video based features like video calling and so on. In short, internet is been helpful to Android for all real time applications as mentioned.

\subsubsection{Bluetooth}

Bluetooth is a wireless technology which is helpful in exchanging the data for approximately 10meters. This exchange of data can be handled in much secure way using the facility called pairing. In pairing, both the devices which are meant for exchange of data is paired with a unique code which is known only to those two devices. Android also supports the facility of Bluetooth by using the Bluetooth option available in the mobile phone [12]. In our project, the microcontroller which has Bluetooth, is paired with the android mobile phone in order to make communication and to send signal from controller to android mobile.

\subsubsection{Touch screen}

Touch screen has become the basic facility of any smartphone. This facility makes the mobile users to access the options of the phone in much easier and faster way. Touch screen helps to swipe, flip or zoom any button on the phone. Android supports the touch facility and latest android phones also support multi touch feature where the user can touch the screen with more than one finger. This facility helps the android to customise the application icon anywhere on the screen. The UI becomes more user friendly where the design of the icon is completely developer's choice. 
Apart from these, Android also supports multiple language support (it supports many language spoken and understood by human), Connectivity (Android can be used for any kind of connectivity technologies like GSM, CDMA, Bluetooth, Wi-Fi, WiMAX, UMTS), Additional hardware support (Hardware features are like magnetometers, gyroscopes, camera, touchscreen and so on), voice based features such as calling which includes both video and non video calls, messaging, voice mails etc.[13].

\subsection{Components of Android operating system}

Android operating system is subdivided into four layers and they are applications, Application Framework, Libraries, Android Runtime (both in one layer) and Linux Kernel as shown in figure $2[13,7,14]$. Applications and Application framework is written in Java and it is upper most layer. Libaries and Dalvik Virtual Machine forms the middle layer and it is written in $\mathrm{C} / \mathrm{C}++$ Language. The bottom most layer is the Linux kernel and it is written in $\mathrm{C}$ language. This Linux operating system forms a shield to Android which helps to wrap the application inside. Developers and the users have access and visibility only to application and application framework. This application written in Java comes in contact with Dalvik Virtual Machine which converts this Java to $\mathrm{C}$ code which is been sent to Linux Kernel for execution of the code. Each layer has its own functionality which is put together to form a complete application for a mobile device.

Applications: The applications are the icon which we see on our mobile device and those Java applications can be such as email, maps, internet, camera, contacts, media, messaging and so on $[7,13]$.

Application Framework: Application Framework is the backbone to the developers. The applications are evolved with the help of API which are available in the framework. This unit is placed to eliminate creating unwanted new components which is already available in the framework. The developers would have complete access to it and hence can reuse and extend the components already present in the API. For example; ActivityManager helps to manage the things which a user does in a particular time. It keeps track of all the activities running in the system and enables proper management of them. Notification Manager helps 
to display the alerts of all the applications on the status bar. Telephony Manager enables to handle the settings of the phone, network connection and also to edit the contact details [7, 13].

Libraries: Android has a set of $\mathrm{C} / \mathrm{C}++$ libraries which helps the developers to create their own application. These libraries are accessible through the application framework and those can't be accessed directly. Different types of libraries are available such as standard C library like libC, SSL, SQLite, Web libraries like webkit which is used for web browser, libraries like open GL|ES are used for graphics, libraries used for audio and video formats like MP3, MPEG4, JPG and AAC are multimedia framework libraries [7, 13].

Android Runtime: Android runtime includes a set of core libraries along with Dalvik Virtual Machine. Every application developed in android have their own process and each of them has their own Dalvik Virtual Machine. Dalvik Virtual Machine is main area in Android platform. Dalvik can run many VMs very efficiently. This VM runs the file in .dex format which helps to minimize the consumption of the memory space of the system. This Virtual Machine runs the class file which is compiled by Java compiler. It converts the byte code to .dex file at compile time using a tool called " $\mathrm{dx}$ " which is embedded in SDK. Developers are able to run multiple applications at same time due to this Virtual Machine [14].

Linux Kernel: Android currently works on Linux kernel version 6. Linux provides many services to the system such as network stack, power management, memory management, security and so on. It also forms as a layer between the software and hardware of the system and helps in their binding which helps in better communication among them [7]. 

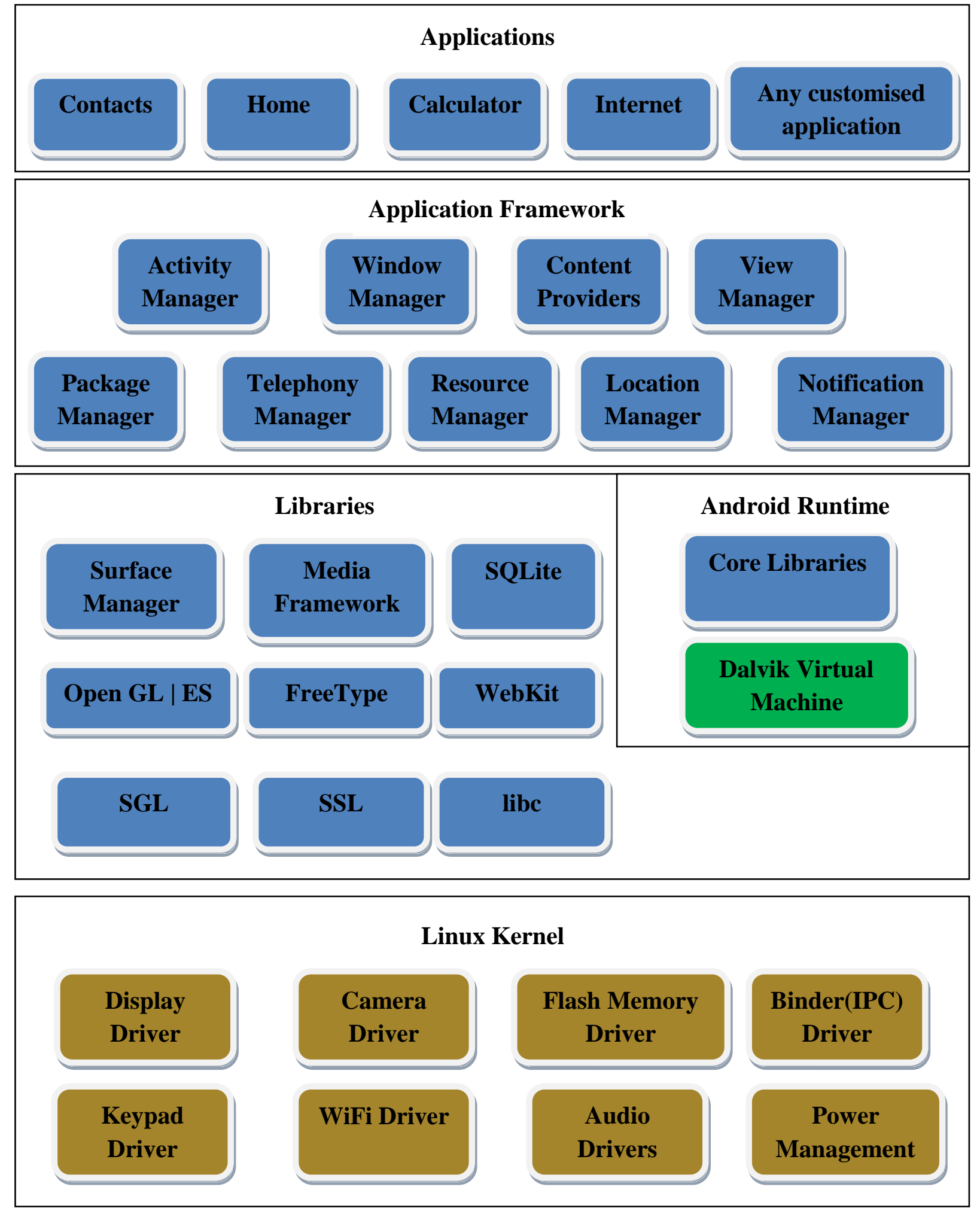

Figure 2 Components of Android operating system 


\subsection{Android Manifest}

Android application evoked from the mobile is been developed in Eclipse and a Java Project is created to do the same. This Java Project forms a hierarchical structure and each folder in it is named by default and each of them has its own meaning and significance. When this project is run in Eclipse, the application is opened. The folder structure is as shown below in the figure 3.

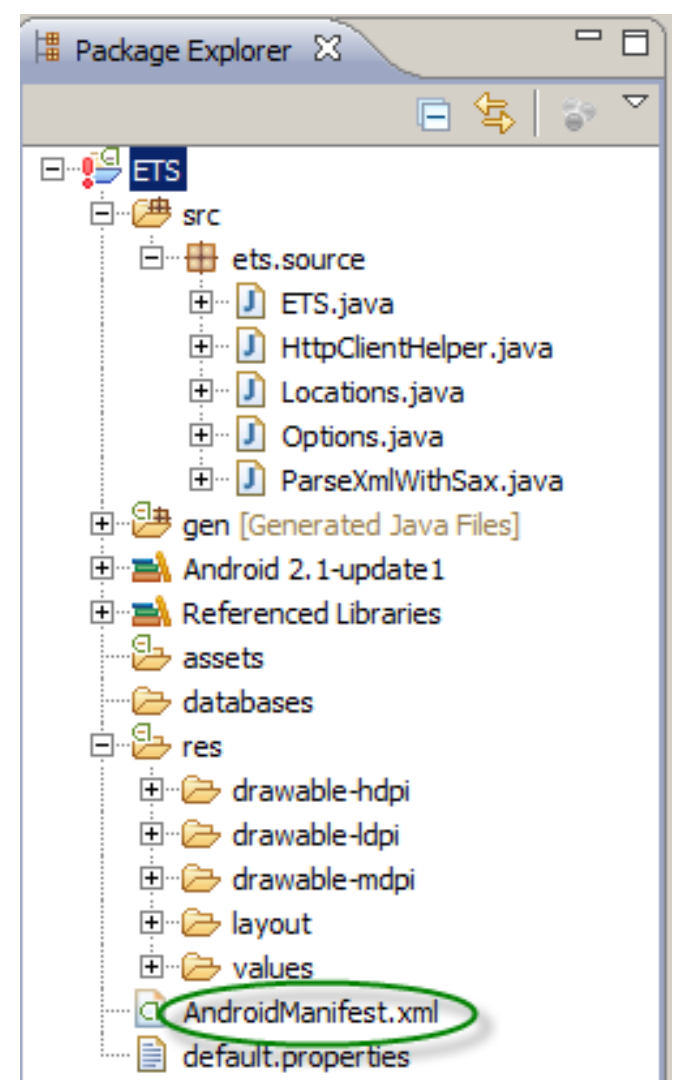

Figure 3 Project Folder Structure in Eclipse

The main focus in this structure is on Android Manifest.xml. This xml file forms the heart of the application. This file tells how the application has to work, when to start and what actions have to be performed. It denotes the main functions and the user permission required for the application. Also this file will set the icon and theme of the application [7]. This xml file has several tags which depend on the action performed by it to the application. Not all the Java Project has same tags in their application. Few Function oriented tag and their significance are described with the help of the sample $\mathrm{xml}$ file given below in figure 4 . 


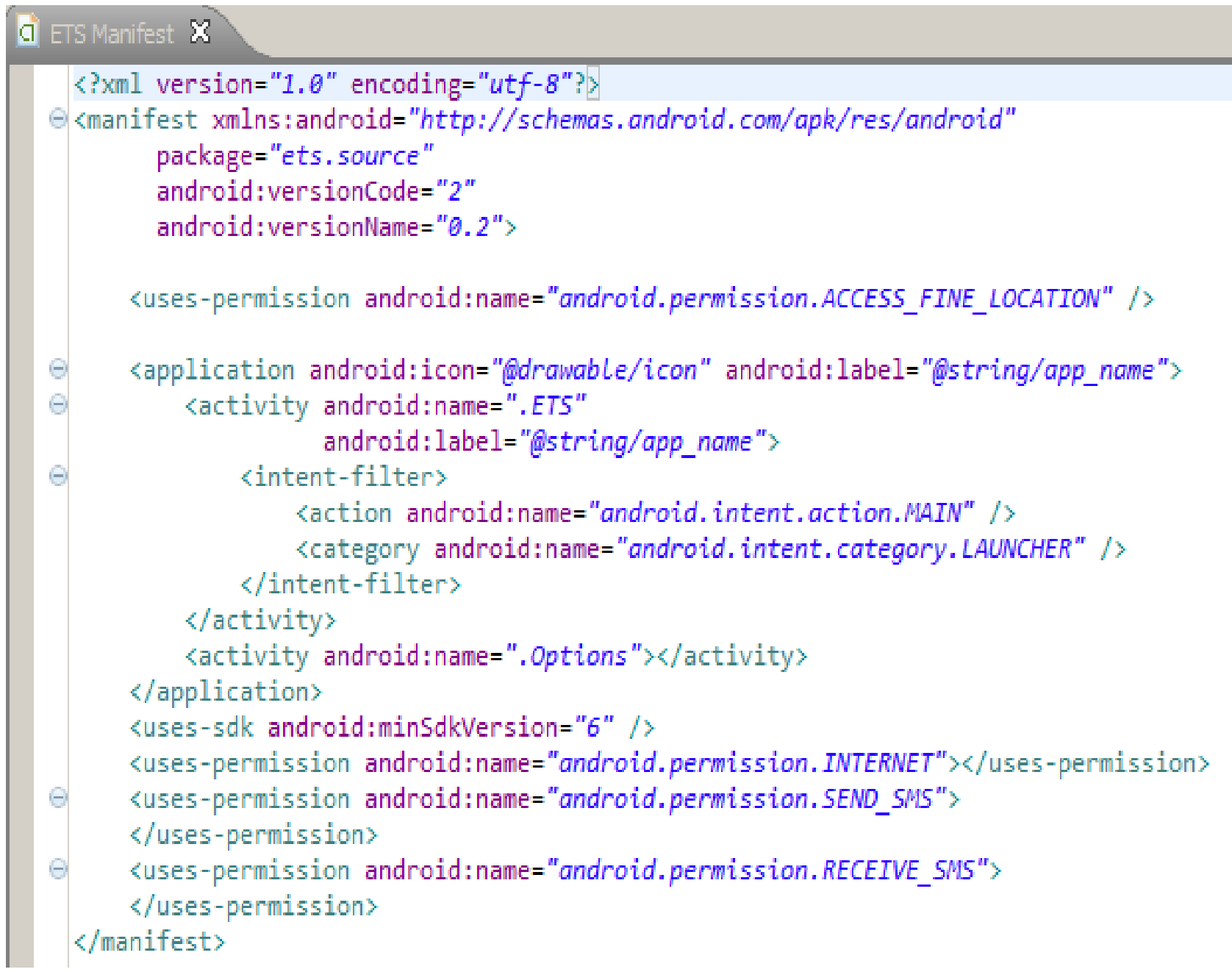

Figure 4 Sample AndroidManifest.xml file

1) Activity - This is a type of $\mathrm{xml}$ tag which shows the display screen of the Android Mobile Phone. It is denoted by <Activity> in the $\mathrm{xml}$ file. For example, the contact list which is displays a screen is an activity, a screen which displays the inbox is an activity and so on.

2) Service - This tag does not require any display function in the Android application. This is denoted by <Service> in the Manifest file. This tag is required in long run for background applications where it is used as service functions. For example, Radio, MP3 Player, Calculator and so on.

3) Intent and Broadcast Receiver - This tag has two different types of tag in the manifest file. Intent denoted by <intent-filter> and Broadcast receiver denoted by <receiver>. Intent tag is 
used to depict the capacity of the parent. It shows what kind of activity or a service function or number of broadcast receivers handled by the parent. This information is shown by intent filter. Broadcast receiver also known as receiver enables the application which accepts the intent. This is been broadcast by the system even when the application is not in running state.

4) Content Provider - It uses <Provider> tag in the manifest xml file. This acts as the database or a storage area where all the relevant information is declared as an element inside this tag provider. Anything which is not declared in this content provider is not visible and can't be executed by the system. For example, the database which the android application uses to store the information, SQLite is a part of the content provider program. 


\subsection{Arduino Microcontroller}

Microcontroller is a smaller version of a computer which has a memory unit, processor and input/output unit. Main advantage of the controller is that it consumes lesser space and makes the design very compact. The significance of microcontroller is that it can be useful for embedded applications where automation of the devices is of at most importance. This feature of controller has encouraged us to use it in our project. There are different types of microcontroller such as AVR, ARM, PIC and so on. Of all these, AVR is the first controller to use flash memory for its storage [15-17]. To suit the requirements such as better sensitivity to the environment, automatic halt of the vehicle, compact design and maximum storage, AVR microcontroller is chosen for our project. Android is an open source platform, hence a controller with similar feature should also be chosen and there comes the choice of using Arduino Microcontroller.

Arduino Microcontroller is an open source prototyping platform which can sense the environment by the sensors which is given as the input to it. The programming of the controller is done using Arduino Programming language. The language used for programming is $\mathrm{C} / \mathrm{C}++$. It consists of an IDE. This IDE is a cross platform application written in Java and then it is derived from IDE for programming and wiring. The best thing about this programming is that the language is so easy to understand that even a beginner can write it easily. IDE also provides sample programs which can be used for further development of the project. Various Arduino microcontrollers are available in market such as Arduino Extreme, Arduino Mini, Arduino Nano, Arduino Bluetooth, Arduino Diecimila, Arduino Duemilanove, Arduino Mega and so on. Each of these microcontrollers have their own significance.Arduino Bluetooth is found as best choice for our project. As the name suggests, this microcontroller has in built Bluetooth module which lacks in other controllers [17].

Arduino Bluetooth (Arduino BT) microcontroller works on principle of Atmega168 and the Bluegiga WT11 Bluetooth module. It has 14 input/output pins and it supports serial communication over Bluetooth. The operating voltage is $5 \mathrm{~V}$ which makes the controller very fragile and hence the voltage should not be exceeded else it would result in the damage of the microcontroller. It has $16 \mathrm{~kb}$ flash memory for the storage of the code. The reset option is at pin number 7 which is connected to the reset of bluegiga WT11 module. The Bluetooth communication is provided by Bluegiga WT11 module on Arduino BT which can connect to 
any devices which has Bluetooth connectivity. It should be configured and should be detected by the device to which it is connected. It works on the baud rate of 112500 . The controller is connected to another device by pairing and the name of the device suggested by Arduino is ARDUINOBT and the passcode is 12345 . This is the default setting of the device [17]. Arduino Bluetooth microcontroller is connected to the Android mobile device via Bluetooth. The challenge is the compatibility of the microcontroller to the mobile phone enabled with android. The programming of the android mobile device should work well with arduino BT microcontroller which is proper integration of them. This amalgamation of the components is done using Amarino.

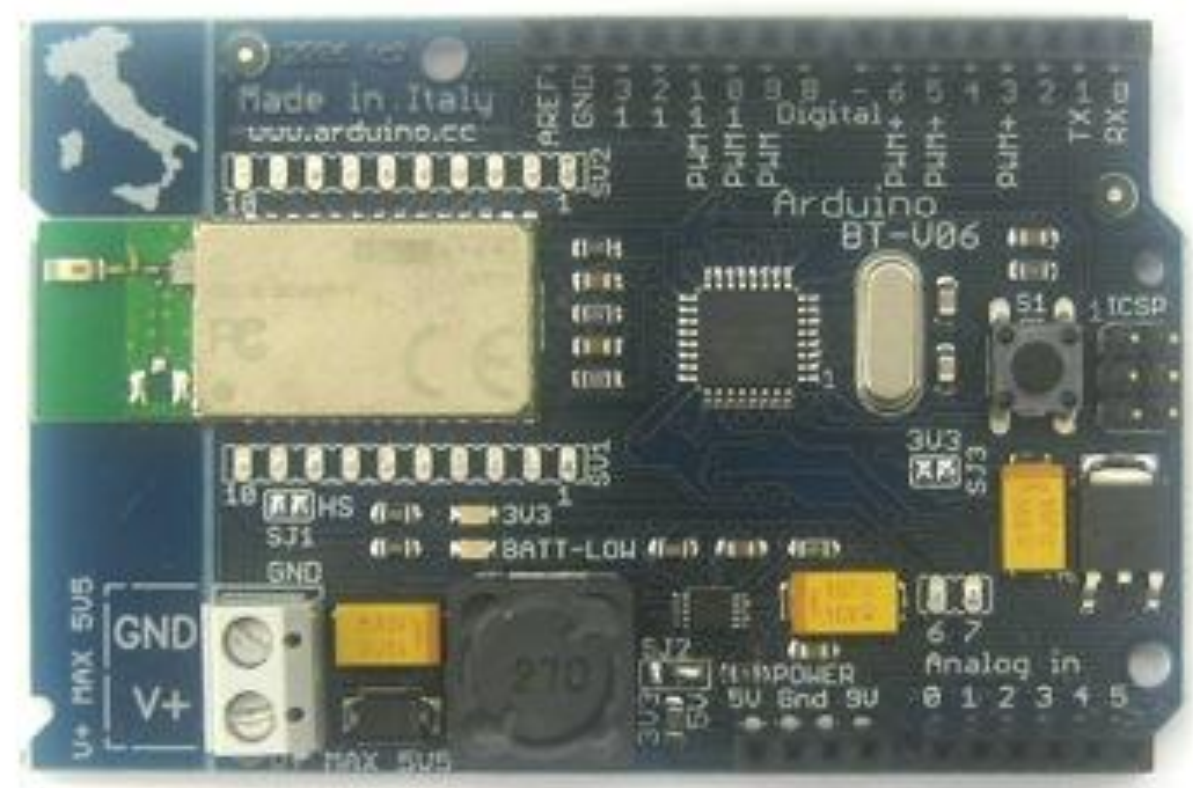

Figure 5 Arduino BT Microcontroller 


\subsection{Integration of Arduino with Android-Amarino}

Every request sent has its own response. The same is the case with a mobile phone. For instance, a phone call is alerted to the user by a ringtone, a text message received is displayed on the screen, a photo clicked with the help of the camera is saved in memory of the phone and so on. These events are generated on the phone itself. The same event can also be viewed somewhere else like in our room, through a sensor like accelerometer or on a microcontroller. To such a situation to occur, Amarino is used.

Amarino is a tool kit which helps in integration of android with arduino. It consists of the Android application and libraries required for arduino. Amarino helps to connect a mobile device enabled with android and an arduino microcontroller via Bluetooth [18].

\subsection{Heart Rate Monitoring Device}

Heart is the main organ in a human's body. One can't live without it. It's because of this anything in the world is compared to heart, that's the importance of such a vital organ. Heart rate is an important factor to be considered in a human body. Heart rate tells us how many times heart beats in a minute. It is usually measured by feeling the pulse on any area near the artery. This measure signifies the blood pressure of a person. The blood pressure either low or high is dangerous to health. Hence it has to be kept under control and also by constant monitoring [19].

Heart rate monitoring is an important aspect of a human being. This monitoring is usually done by a regular health checkup at any hospital. This is a normal scenario, but there are situations where the heart rate is not monitored while driving any vehicle or while exercising and so on. Hence a heart rate monitoring device is very essential. Heart rate monitoring is done at any hospitals using devices like ECG. Even though it is accurate, this device is costly and also regular visit to hospital should also be carried out. Also a person with heart disease complaint should be able to monitor his condition continuously. To solve all these criticalities, a heart rate monitoring device has to be purchased and maintained for personal care. These days heart rate monitor is been used commonly by normal person rather than in a hospital [20, 21]. 
Heart rate monitor helps to detect the abnormalities in the heart and would display it to the person who is using it. This feature has inspired us to use such a device for our emergency conditions especially when a person is driving a car and is suddenly met with heart attack. To get situation under control, this monitor device would send an alert to the android enabled mobile phone which will in turn halt the car to avoid further causalities. There are various heart rate monitoring device are available in the market such as Zephyr HR Bluetooth heart rate monitor, polar Bluetooth heart rate monitor, Wahoo Fitness ANT plus Dongle and so on [22].

Zephyr HR Bluetooth enabled heart rate monitor is best suited for our project for various reasons such as the Zephyr programming is easier and it is open source. It is a device with Bluetooth connectivity which avoids wired connection and reduces the hardware cost for it. It also has a fabric sensor which detects the data irrespective of any fabric. Speed, distance is also displayed using this device which helps to see a pictorial representation of a person's heart rate. The best thing about this device is that it can tolerate any extreme motion of the body like running, jumping, jogging and so on. Also the transfer of data is via Bluetooth [22].

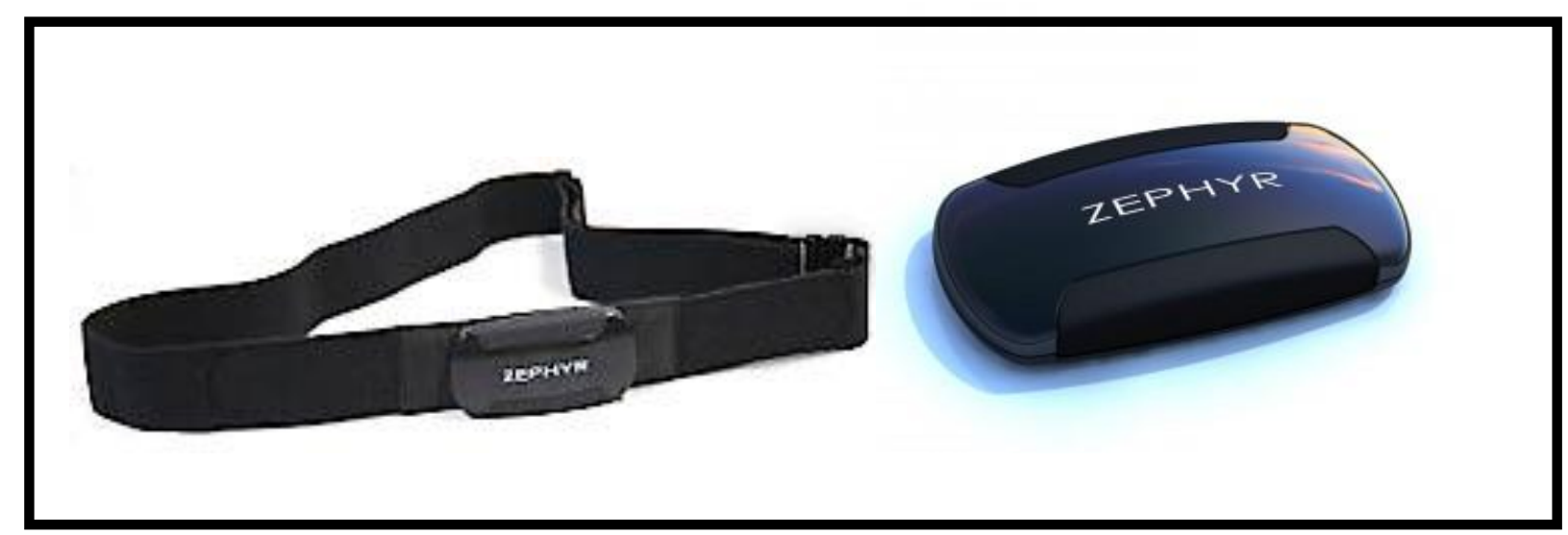

Figure 6 Zephyr Heart rate monitoring device 


\section{CHAPTER 3}

\section{Research Methodology}

Our main goal of this project is to track the location of the person in emergency situations where there is no user interaction and send that location information to a pre-stored number.

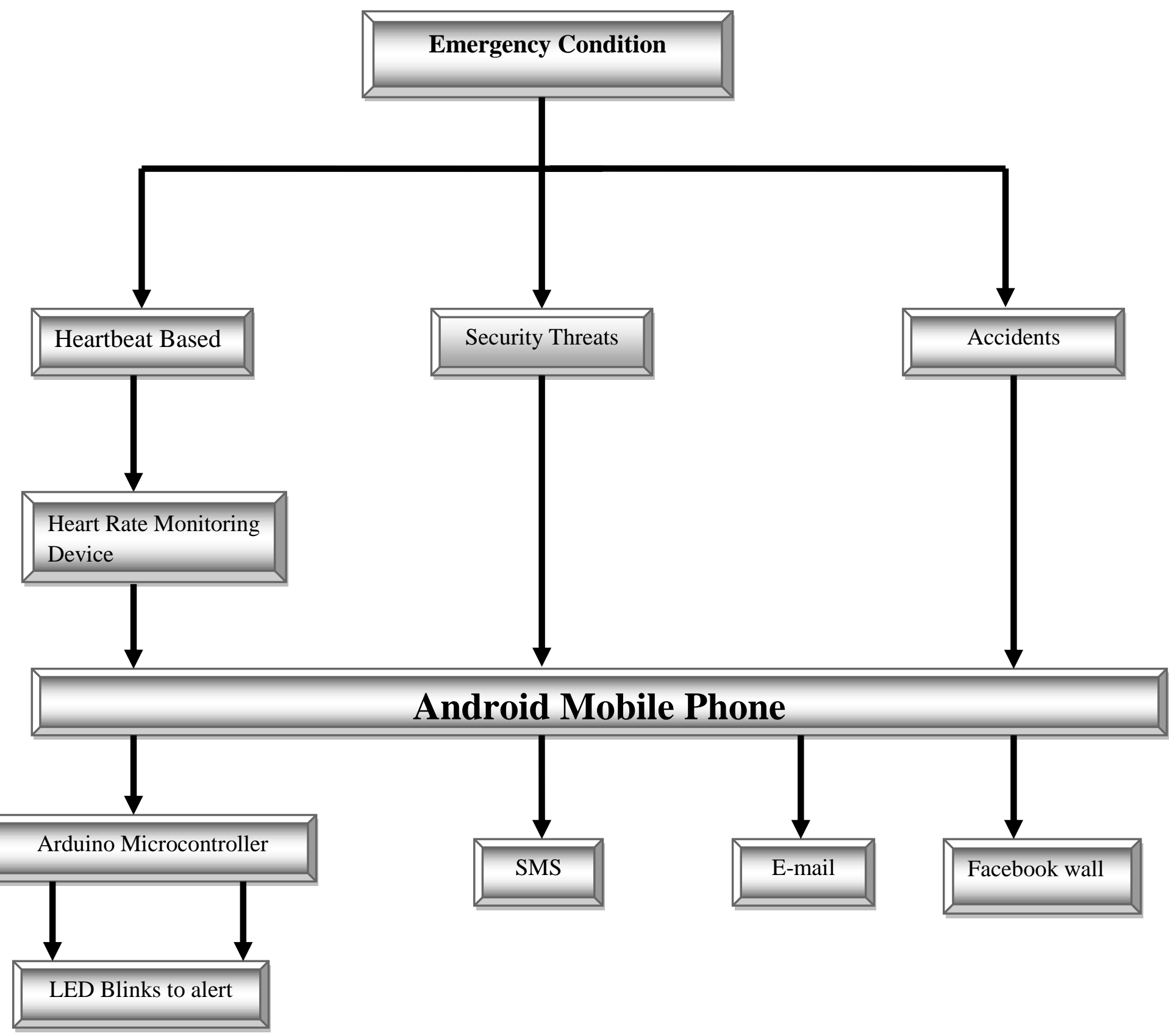

Figure 7 Emergency Condition Scenarios 


\subsection{Research Questions}

1. How can the location of a person be tracked and notified using Google's Android platform in case of Emergency?

2. How to monitor the heart rate of a person and to manipulate those data to detect a critical situation using Android mobile platform?

3. How to integrate Arduino microcontroller with android mobile device?

\subsection{Expected outcomes}

The expected outcomes of our thesis are

- The exact location of a person will be tracked using GPS and location information will be sent to a pre-stored number via SMS, email and message will be posted on Facebook wall in case of emergency.

- Heart rate of a person will be monitored and will be notified under critical situation to android mobile device and using that information the location of a person will be tracked and will be sent to an emergency contact number/email/Facebook wall.

- Under critical situation of heart rates, the android mobile send a signal to a microcontroller and LED in that microcontroller blinks to make an alert of risk signal.

\subsection{Main Contribution}

- To design an application for android enabled mobile device and to track the location of a person using GPS and to send the location information to a pre-stored emergency contact number.

- Design an application and user interface using Java program to integrate SMS functionality to send geographical information to another remote emergency email address and also message which has to be posted on Facebook wall.

- Integrating Ardunio microcontroller with android mobile phone, to make an alert of risk signal under critical situation. 


\section{CHAPTER 4}

\section{PROBLEM SOLUTION}

\subsection{Heart Rate Based}

\subsubsection{Problem Statement}

Nowadays, mobile devices started to integrate with various third party hardware's to provide more functionality to the users, which also leads to the integration of a heart rate device which will monitor the heart beat of an user. But how the heart rate device can be integrated with Android mobile, so that the android enable mobile can monitor the heart beat of a person, and also how to use that heart rate to manipulate a person under emergency? The Zephyr heart rate monitoring device is used to fetch the heart rate of a person and that device is integrated with the android mobile with help of programmable application, developed using android SDK and, this application will decide about the critical situation with respect to heart rate and sends a message to a pre-stored emergency contact number which also contains the geographical location of the user.

\subsubsection{Scenario}

The design of this project deals with a person/user driving in a vehicle. A typical scenario is, when a person driving in isolated roads, wearing the Zephyr heart rate device around the chest. This heart rate device will send the heart rate every second to the android mobile via Bluetooth by which it is monitoring the heart beat of the driving person. The heart rate is normal between 60-100, if it is less than 60 it's called has Bradycardia and if it's more than 100 it is called as tachycardia. In most condition the heart beat becomes less when there is dehydration, decreased protein intake and it becomes more in uncontrolled hypertension. 


\subsubsection{Modeling}

Our application is designed to sense this heart rate, and if there are any abnormalities in the heart rate like, if the heart rate goes below 60 or above 100, automatically the android mobile will send a signal to an Arudino microcontroller which is connected to android mobile via Bluetooth. This Arduino microcontroller will make an alert signal, in our case the alert signal is indicated by blink of a led. Simultaneously our application will track the location information of the user who is under emergency and send that location information to a remote pre-stored emergency contact number. This scenario is shown in figure 8.

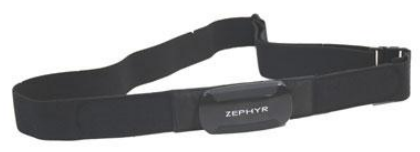

2. Zephry Heart Rate device sends heart rate to mobile

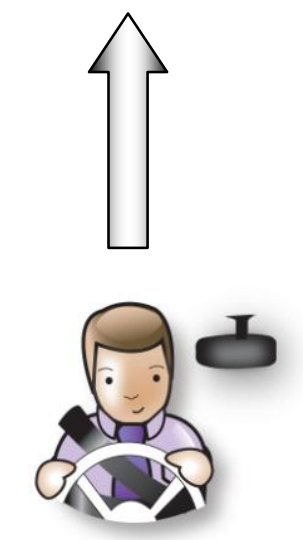

1. Person wearing the Heart rate device while driving
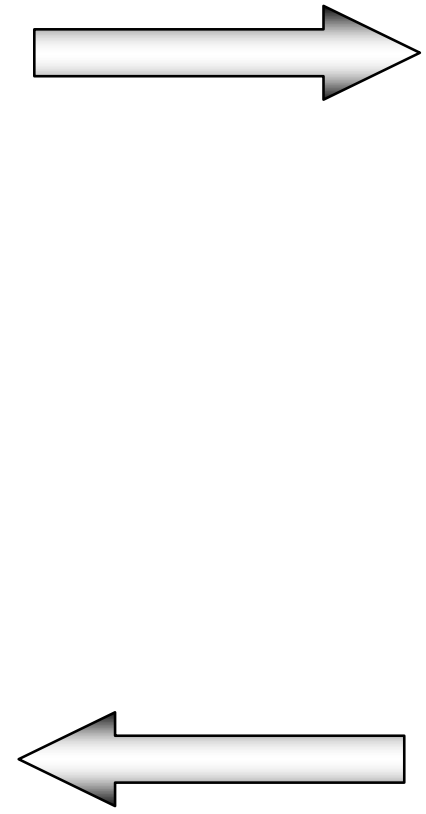

Figure 8 Modeling of heart rate based scenario
4. Arduino BT getting signal from android mobile \& shows alert signal
3. Android Mobile with ETS

application, which gets heart rate data and sends a SMS under emergency

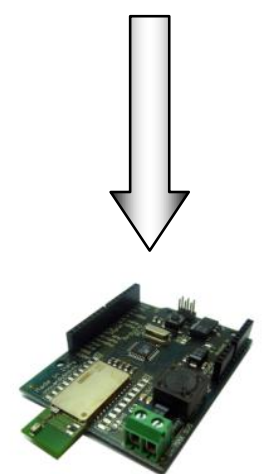

$$
\text { android mobile \& shows alert signal }
$$




\subsubsection{Implementation and Validation}

\subsubsection{Implementation of API with Heart Rate Device}

Implementation of communication between a zephyr heart rate (HR) device and android mobile starts with designing an API. Figure 9 below shows the Zephyr Bluetooth communicates with a mobile device over the Bluetooth link. The Zephyr Bluetooth HR device uses a Bluetooth SPP (Serial Port Profile) to communicate with the low level protocol such as

$>115,200$ baud rate

$>8$ data bits

$>1$ stop parity bits

$>$ No parity

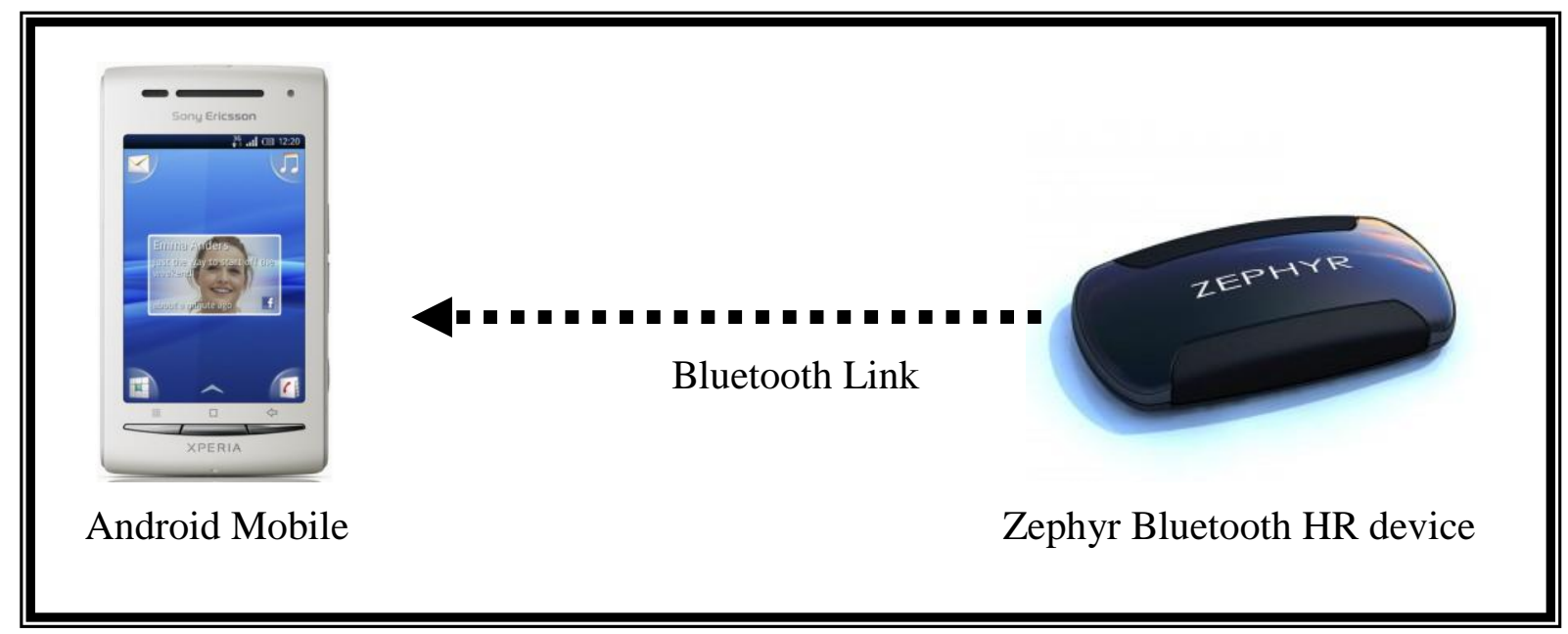

Figure 9 Communication between Zephyr HR device and Android mobile phone

Our application employs the API and enables the HR device to transmit the different packet types such as heart rate, speed and distance packets. The following steps below are a description of the most important aspects of the source code in our application used to enable the General Packet and display the data on the Android phone.

1. On clicking the Connect button, a Bluetooth adaptor type object is created and passed to an object of the BT Client class type. The BT Client object is essentially a thread that manages the overall Bluetooth connectivity of the phone with the HR device. 
2. Next, an object of the NewConnectedListener class will need to be created which eessentially implements the ConnectedListener interface, and one that extends the ConnectedListener class. This object is responsible for reacting differently to different kinds of messages. In this object we override the parent class's connected method and define our own method. In this method we create a ZephyrProtocol object and call its addZephyrPacketEventListener method. This method takes a ZephyrPacketListener argument, in whose ReceivedPacket method we define what message we are interested in, and how we want the data to be displayed on the phone screen.

3. This ConnectedListenerImpl object needs to then be connected to the BTClient object type via addConnectedEventListener function call to tie this object to respond to a received packet from the HR device.

4. Calling the start function of the BTClient thread kicks off the communication of the Application with the HR device. 


\subsubsection{HR device communications Link}

Once the Bluetooth connection has been established with the HR device, the data packets can be received as shown in figure 10

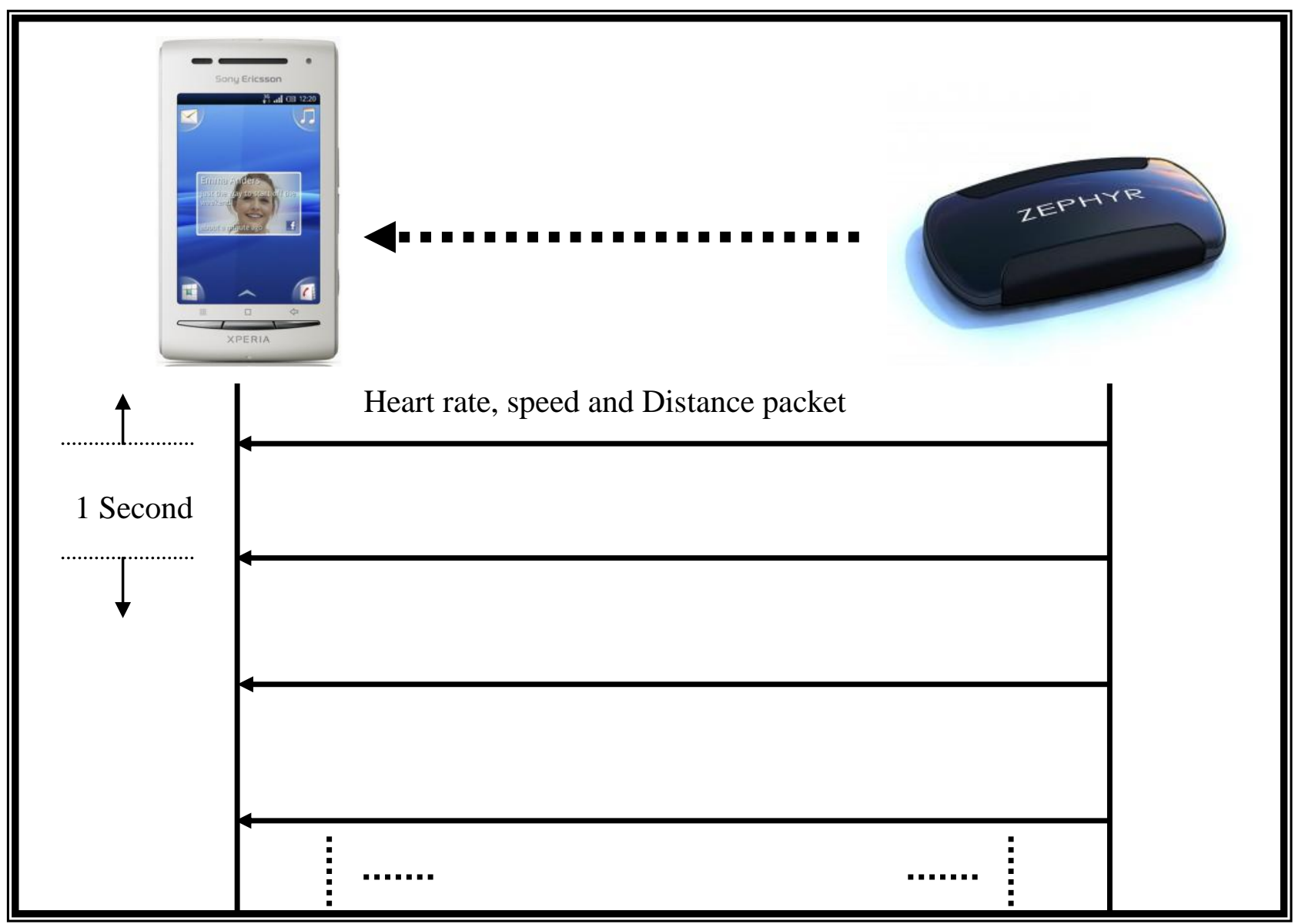

Figure 10 HR device communication link

From the above figure 10, it shows that, when the mobile device is connected to the HR device, mobile receives a data packet from the HR device at 1 second interval. Heart rate is an unsigned byte, constituting the heart rate beats per minute. The effectual range is 30 to $240 \mathrm{bpm}$, but the value will go to zero if no valid heart beat is detected within the timeout period. Heart beat number is an unsigned byte, which is incremented each time a heart beat event is detected, the valid range is $0-255$. This enables the receiver to determine how many heart beat timestamps are present in the received packet, even in the event of a dropped packet. 


\section{Validation:}

The HR device powers on automatically when worn. If there is insufficient skin conductivity (excessively dry skin and/or strap sensor pads), the wear-detect circuitry may not trip. Moisten skin and sensor pads with water. The heart rate device has to be worn around the chest region just near the sternum as shown in the figure 11 .

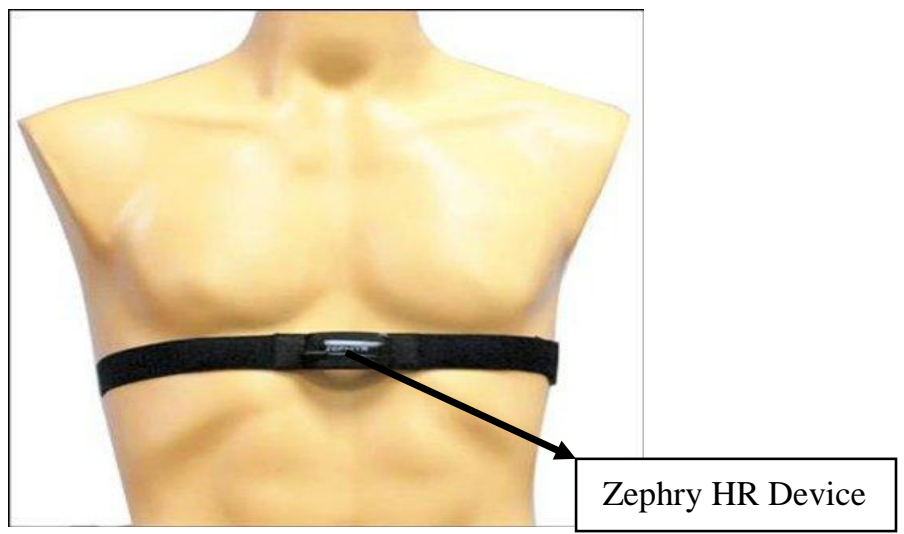

Figure 11 Zephyr heart rate device worn

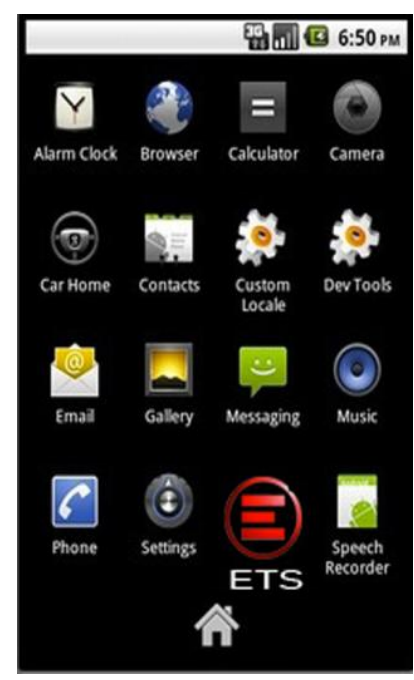

Figure 12 ETS Application

Open ETS-Emergency Tracking System application which is installed in the android mobile as shown in figure 12. As soon as the ETS is clicked, home page with menus such as Pair HR Device, Pair Controller, Enter Details, Start and Disconnect is opened. In order to get the heart rate, user need to pair the HR device with the android mobile. As soon as the HR device is paired with the mobile, the heart rate starts to get displayed on the mobile screen. The entire workflow can be seen in the figure 13.

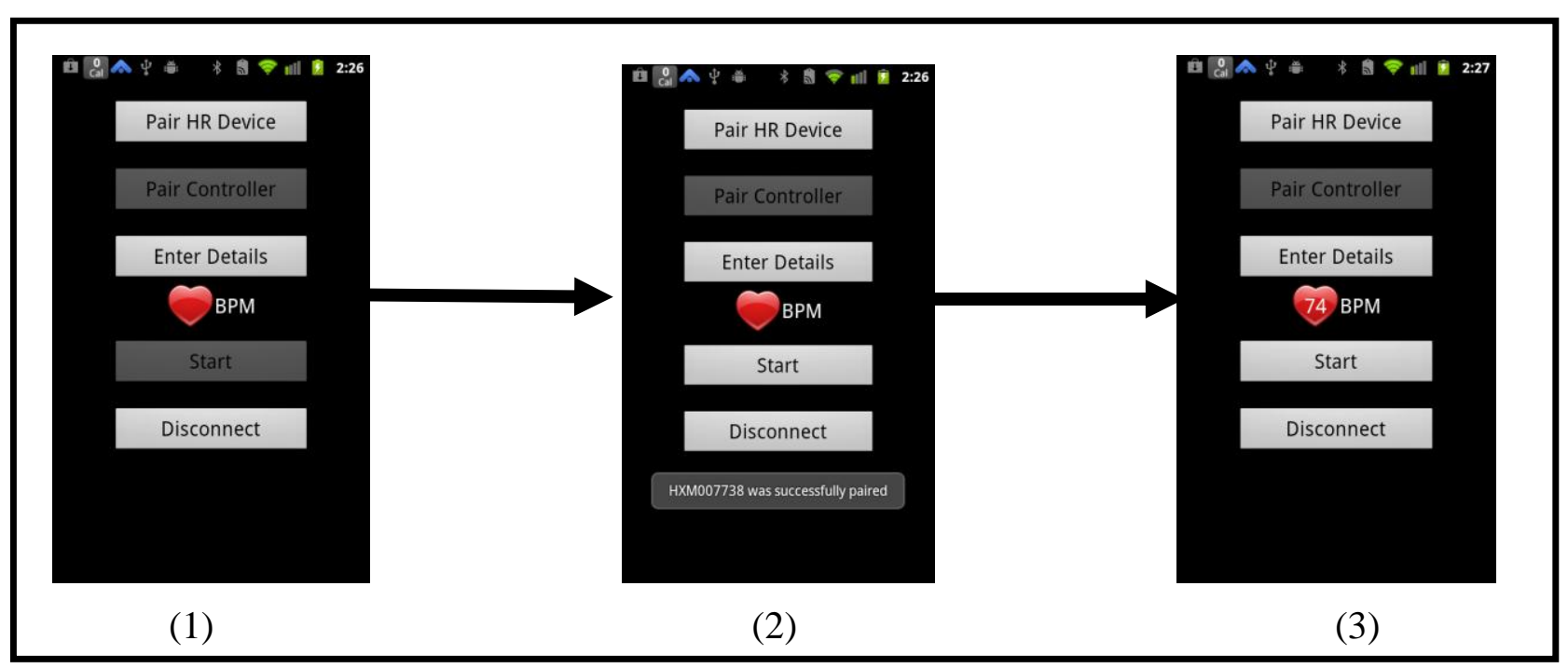

Figure 13 Entire Workflow of Pairing HR device to Android phone 
1. Connects to HR device by tapping on "Pair HR Device"

2. A message shows HR device is successfully paired

3. Heart rate get displayed on the screen "74 BPM (beats per minute)"

The emergency contact number, email address and Facebook account can be integrated in the "Enter Detail page" in the main menu of ETS as shown in figure 14

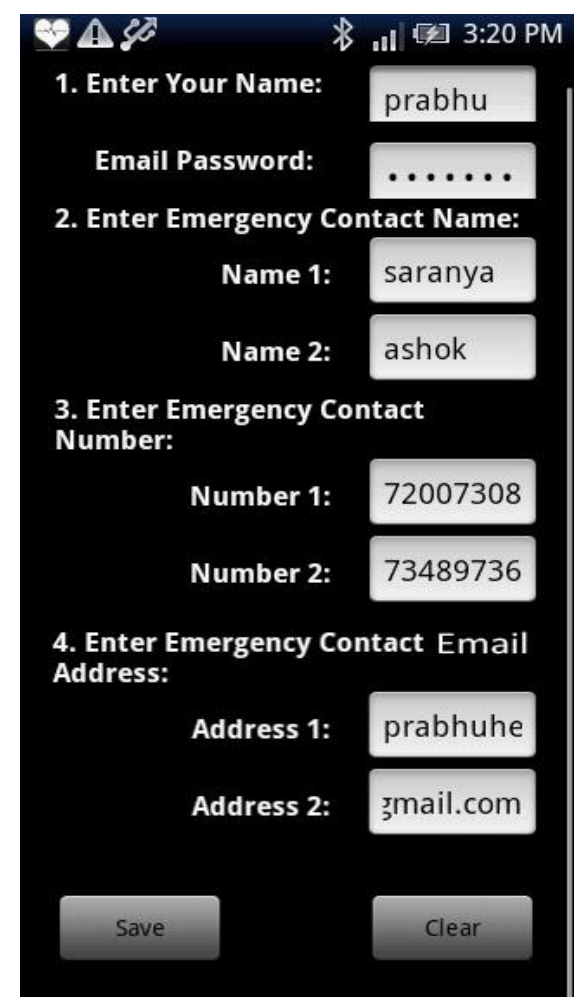

Figure 14 Enter Details page 
When HR device starts to transmit the heart rate to mobile, ETS application performs condition check with respect to $60<\mathrm{HR}<120$, if heart rate is less than 60 or more than 120 then the application decides that this condition is critical and starts to track the location of the user using GPS API and simultaneously sends a message to pre-stored emergency contact number and also to a pre-stored Email address. Finally a message containing "This person is under emergency take necessary action" followed by the geographical location of the person is posted on the enabled Facebook wall. Simultaneously an alert signal is send to arduino microcontroller, to acknowledge the risk signal a LED is connected to pin 13 of the microcontroller and that LED blinks under critical situation. The entire work flow is represented in figure 15.

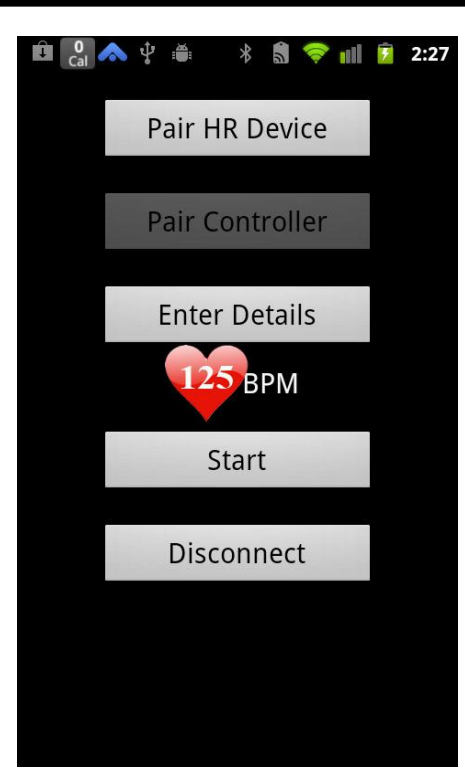

(1)

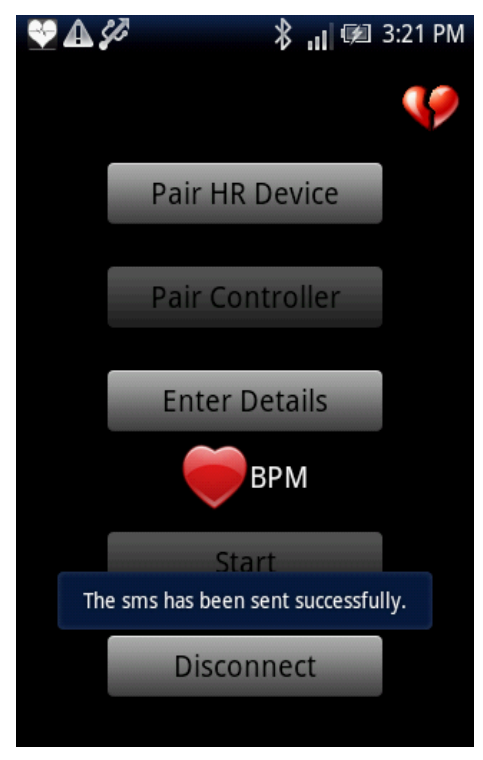

(2)

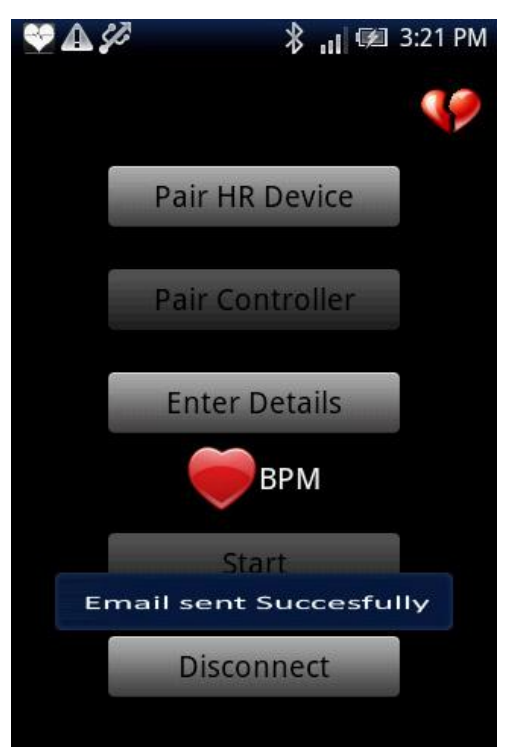

(3)

Figure 15 Abnormal heart rate scenario - workflow

(1) ETS reads an abnormal heart rate of 125BPM

(2) SMS was sent to a pre-stored number

(3) Email was sent to a pre-stored Email address 


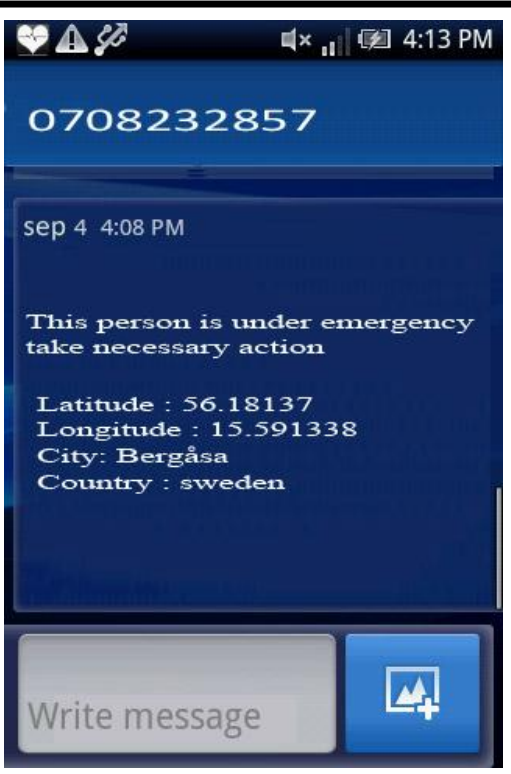

(1)

\section{EMERGENCY!!!}

altair8624@igmail.com Add to contacts

To myworld0501@gmail.com

Person is under emergency condition take necessary action

Latitude : 56.18137

Longitude : 15.591338

City: Bergåsa

Country : sweden

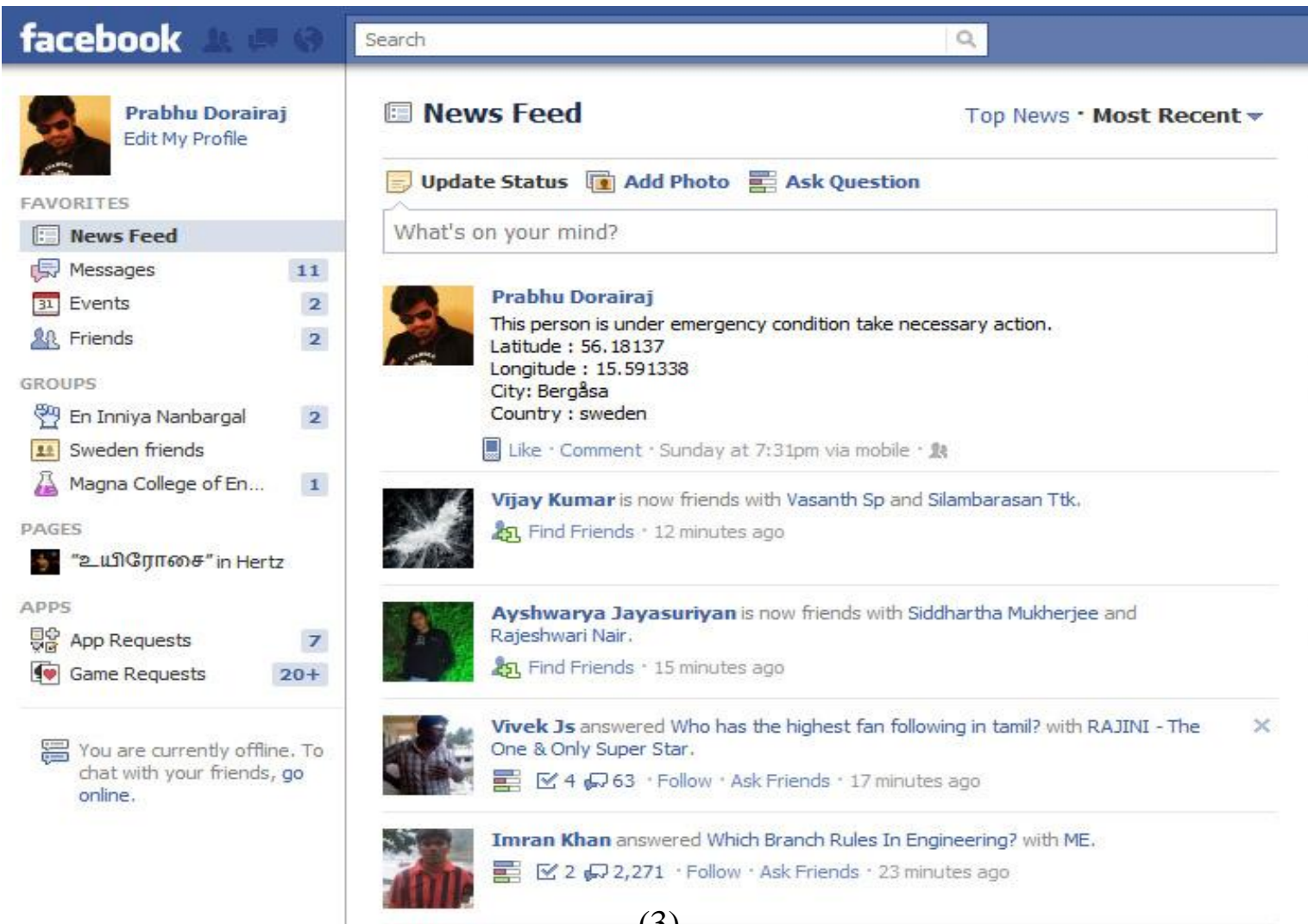

(3)

Figure 16 Message sent via SMS, E-Mail and Facebook Wall

(1) Message received with GPS location as SMS

(2) Email received with GPS location

(3) Risk message was posted on Facebook wall of the respective person under emergency 


\subsection{Security Threats}

\subsubsection{Problem Statement}

The use of GPS on mobile devices in the recent generations of mobile communication is one of the ubiquitous applications that are effectively developed. Symbian is the most utilized mobile operating system in this regard that most mobile devices make use of. Window's mobile, Apple's iPhone, Blackberry and others also have their own versions. But how can the location of a person be tracked and notified using Google's Android platform? The GPS Application Program Interface (API) is used to fetch the latitude and longitude coordinates and these are used by SMS manager to send the information as a SMS to a friend or emergency contact which is a pre-stored number. Thus, the rich application features of the Android like the GPS API and SMS manager can be used to track and notify.

This project is developed with Java programming language which is executed on eclipse IDE and implemented on Android Mobile Platform in turn the validation will be performed using an emulator. A simple graphical user interface is used to manage the scenario and make it eventually available to the public at large.

\subsubsection{Scenario}

Security threat can occur anywhere at any time. This project is designed to overcome personal security threat under user request. If the user is in emergency and if the user feels that his/her location has to be sent to a remote user then this project comes in to action by providing a exact geographical location information, which can be sent to pre-stored number and to E-mail address under user request.

\subsubsection{Modeling}

The model of this project is presented in figure 17. It depicts the working model of this project to show the location-based mobile service design on Android and the SMS functionality as well. In order to fetch the current position of the user it is needed to obtain the longitude and latitude coordinate values. All the applications should carry an AndroidManifest.xml file (with precisely that name) in its root directory, since the essential information about the application will be held by the manifest. Hence we can access the 
protected part of APIs. To expend the GPS functionality, we add ACCESS_FINE_LOCATION to get permission to Androidmanifest.xml. To obtain the coordinate values, a location manager has to be created. Location manager is the part that is responsible for creating a location based service on Android. The snippet code below shows how the location manager can be created

\section{LocationManager $\operatorname{lm}=($ LocationManager $)$}

getSystemService(Context.LOCATION_SERVICE).

After fetching the longitude and latitude coordinate values from GPS, a SMS message which contains the location information is generated. In order to send a SMS message, SEND_SMS permission is added to the AndroidManifest.xml file which in turn makes the application to send SMS message using sendSMS() function thereby initiating the smsManager to allow the application to send a SMS message.

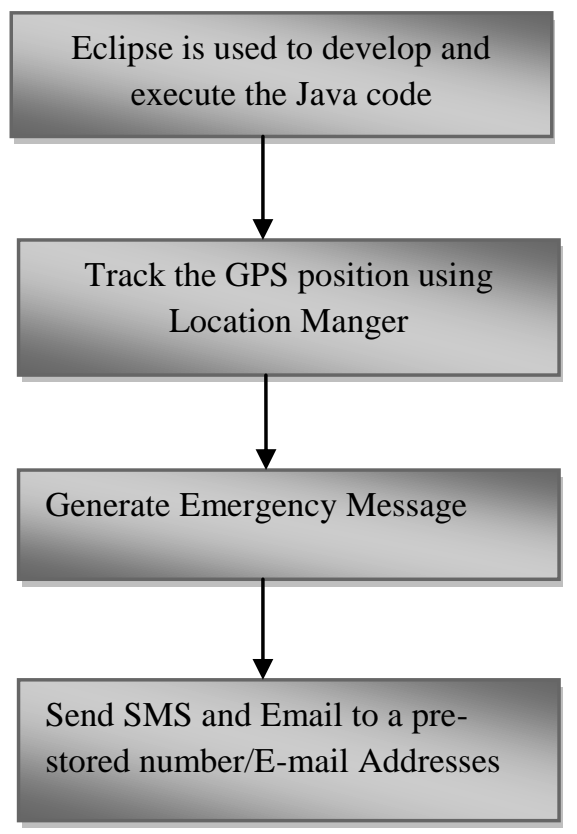

Figure 17 Modelling of Personal safety Scenario 


\subsubsection{Implementation and Validation}

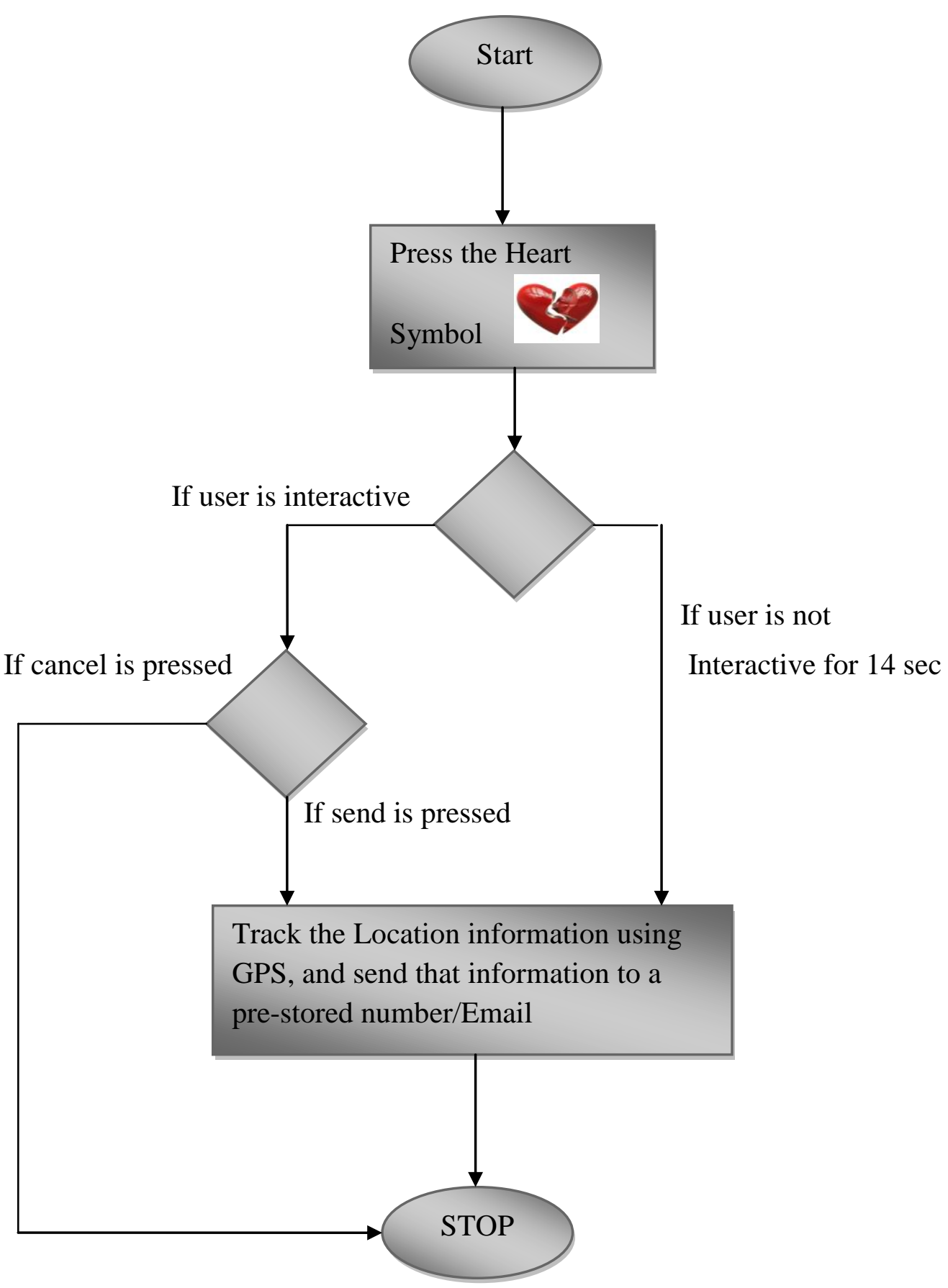

Figure 18 Implementation of Personal Safety scenario 
When the Heart symbol button is pressed on the ETS home page as shown in figure 18 , the ETS application senses that user is under security risk and waits for the user to press the send button. In case the user is not interactive to press the button after pressing the Heart symbol, the system automatically counts down for 14 seconds and then starts to get the location information which contains the latitude and longitude coordinates using a GPS API. Subsequently, the latitude and longitude coordinates are converted into city and country location information as shown in figure 16, this is sent via SMS, email and along with the message "This person is under emergency take necessary action" will be posted on Facebook wall of the registered user. The entire workflow is depicted in figure 19

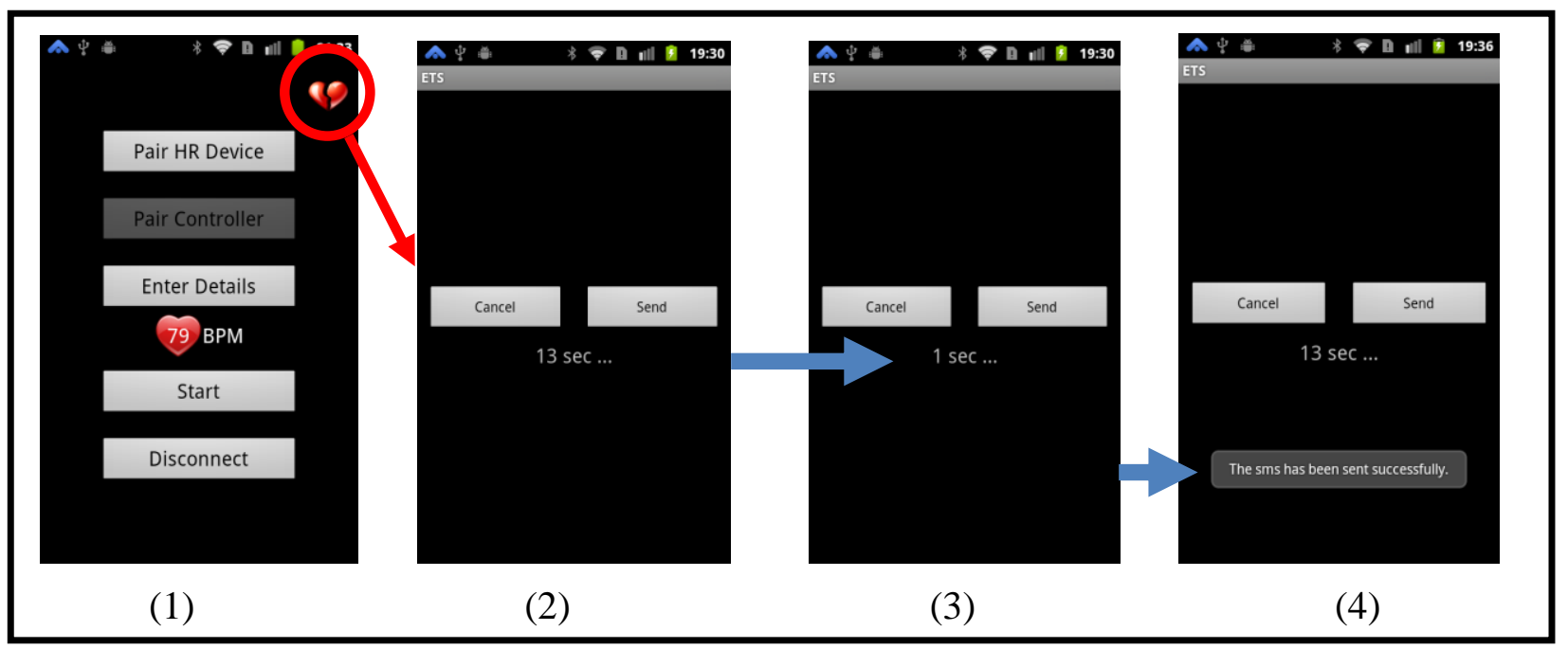

Figure 19 Workflow of Personal Safety scenario 


\subsection{Accidents}

\subsubsection{Problem Statement}

In present world, driving a vehicle has to be done with at most care, otherwise it would result in accident and the reasons behind it could be like negligent driving, drunkard driving, emergency conditions like during heart attacks and so on. The main reason for a person's death during accidents is unavailability of the first aid provision which is due to the delay in the information being reached to the hospital about the accident. The worst case to this problem is the accident occurred while a person is travelling in a vehicle. So how could we track the accident of a person as soon as it has occurred? An in built sensor of the mobile called Accelerometer would help to detect any slight movement of an object and the object being in our case is the mobile phone which is docked inside the car and not being held in hand or in pocket of the person who is driving the vehicle.

\subsubsection{Scenario}

The design of the application is for the purpose of a person who is driving a vehicle. The scenario is, when a person is driving with the mobile phone kept in the car at a fixed position. The mobile should not be kept in pocket or held in hand of the person. Any slight movement of the mobile device would be detected by an in built sensor called Accelerometer.

\subsubsection{Modelling}

Our application is designed using the sensor accelerometer which will detect any tilt in the mobile device. At times this tilt might be just by mistake where the person might have pressed it accidentally. In such a situation the application will wait for $14 \mathrm{sec}$ for the user to enter send or press cancel. If the user doesn't press any key within that time, then the application considers the person to be in danger and sends an emergency alert message to a pre stored number in the mobile. The key assumption of this application is that the mobile phone should not be kept along with the person who is driving the vehicle. This scenario can be represented using a flowchart as in figure 20 


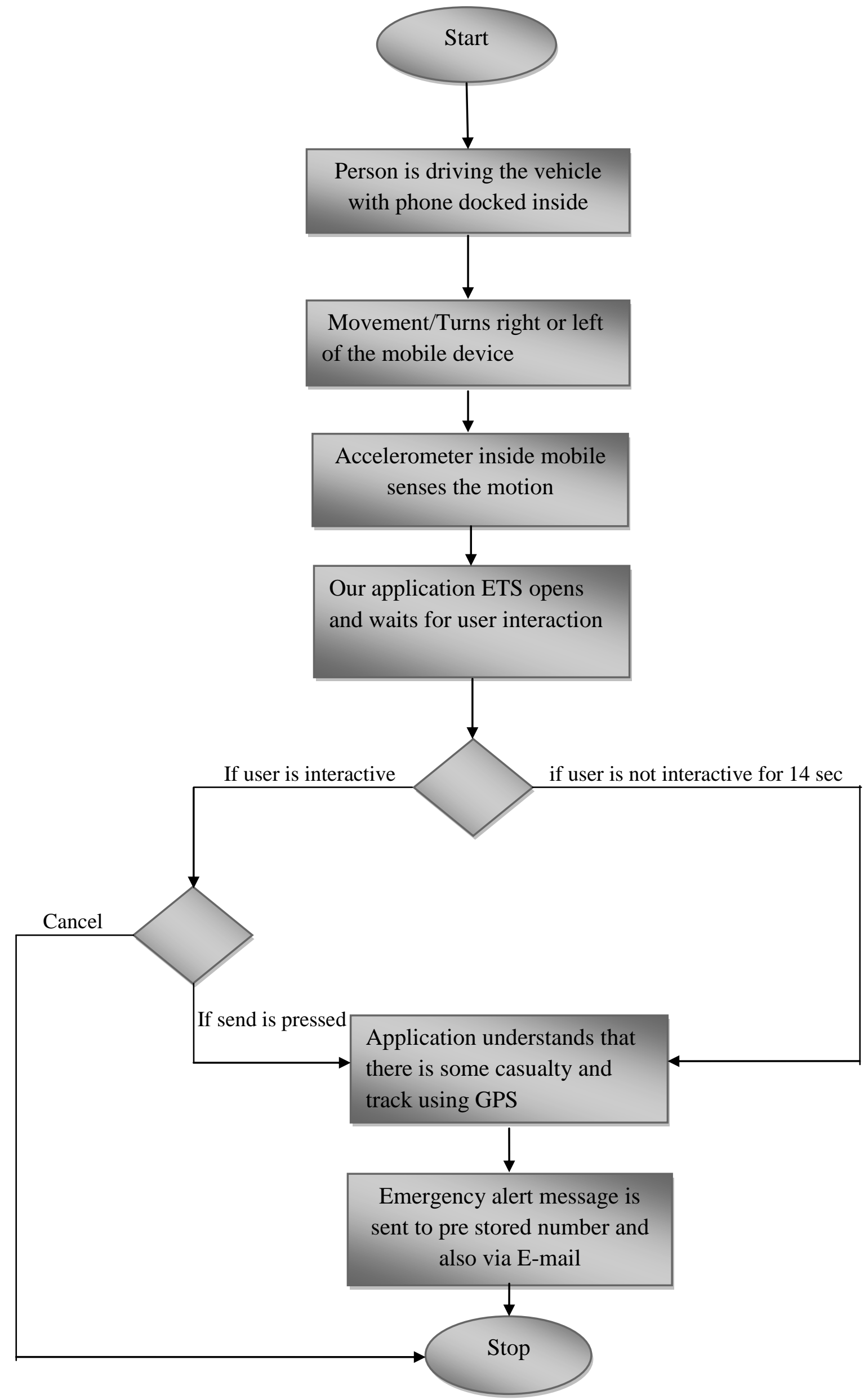

Figure 20 Modeling of Accident scenario 


\subsubsection{Implementation and Validation}

Accidents, which will be sensed by the mobile using a specialized sensor called as accelerometer which is present inside android mobiles. The sensors of these type cadences the acceleration applied to the device. Reckons the device's orientation grounded as shown in figure 21 on the rotation matrix. SensorManager is a public class which lets us to access the device's sensors. Below snippet is the class overview of initializing and activating the accelerometer sensor.
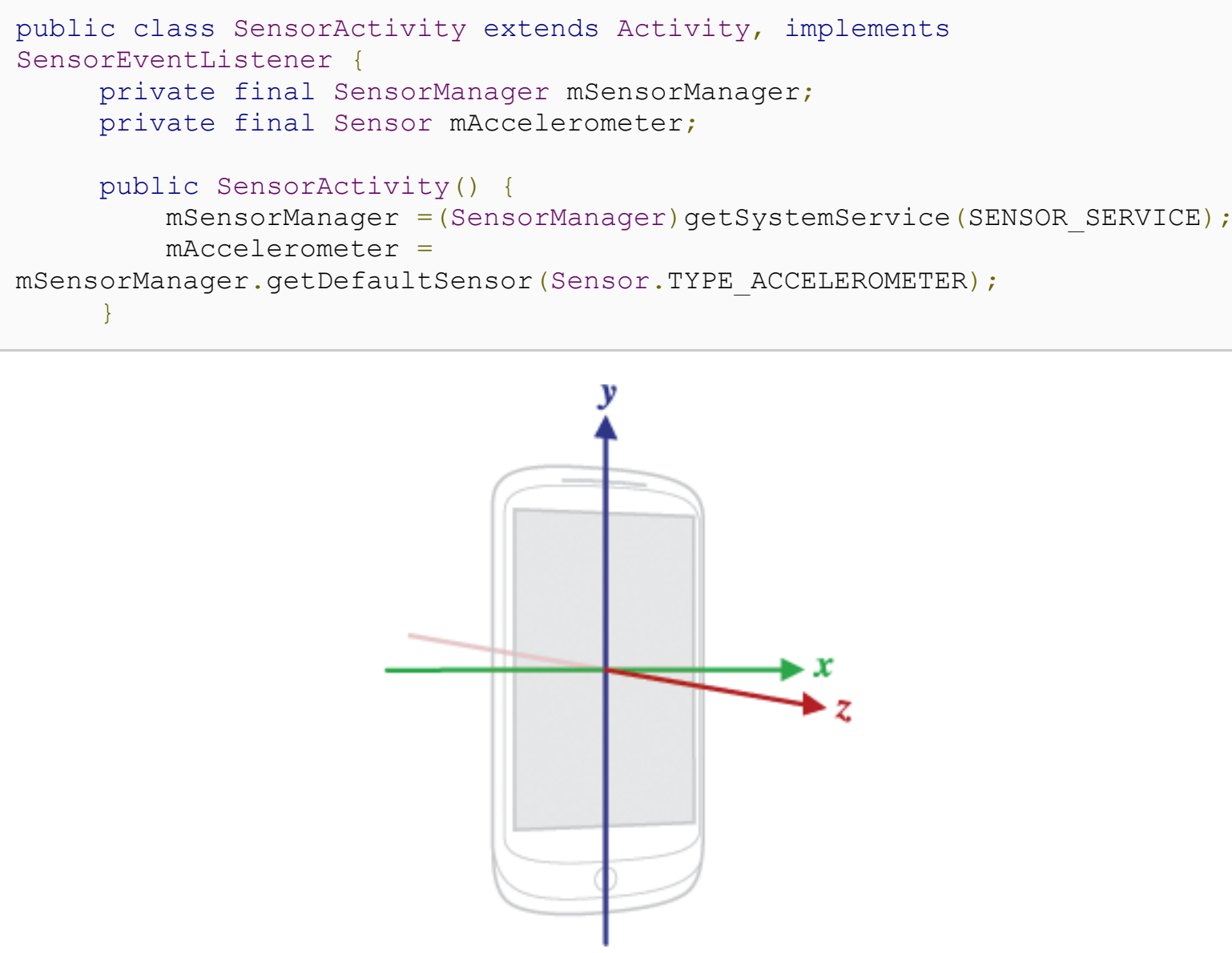

Figure 21 Movement of Mobile

The validation of the accelerometer is performed by tilting the mobile left or right or free fall motion. As soon as the there is a tilt or the mobile orientation then ETS application senses that there is a risk situation and starts to perform the operation of tracking the location information of the user before that, the ETS waits for $14 \mathrm{sec}$ for user to be interactive, where user can perform 'cancel' and send as shown in figure 22. If there is no user interaction then automatically application sends a SMS, Email, and message is posted on Facebook wall as shown in figure 16. 


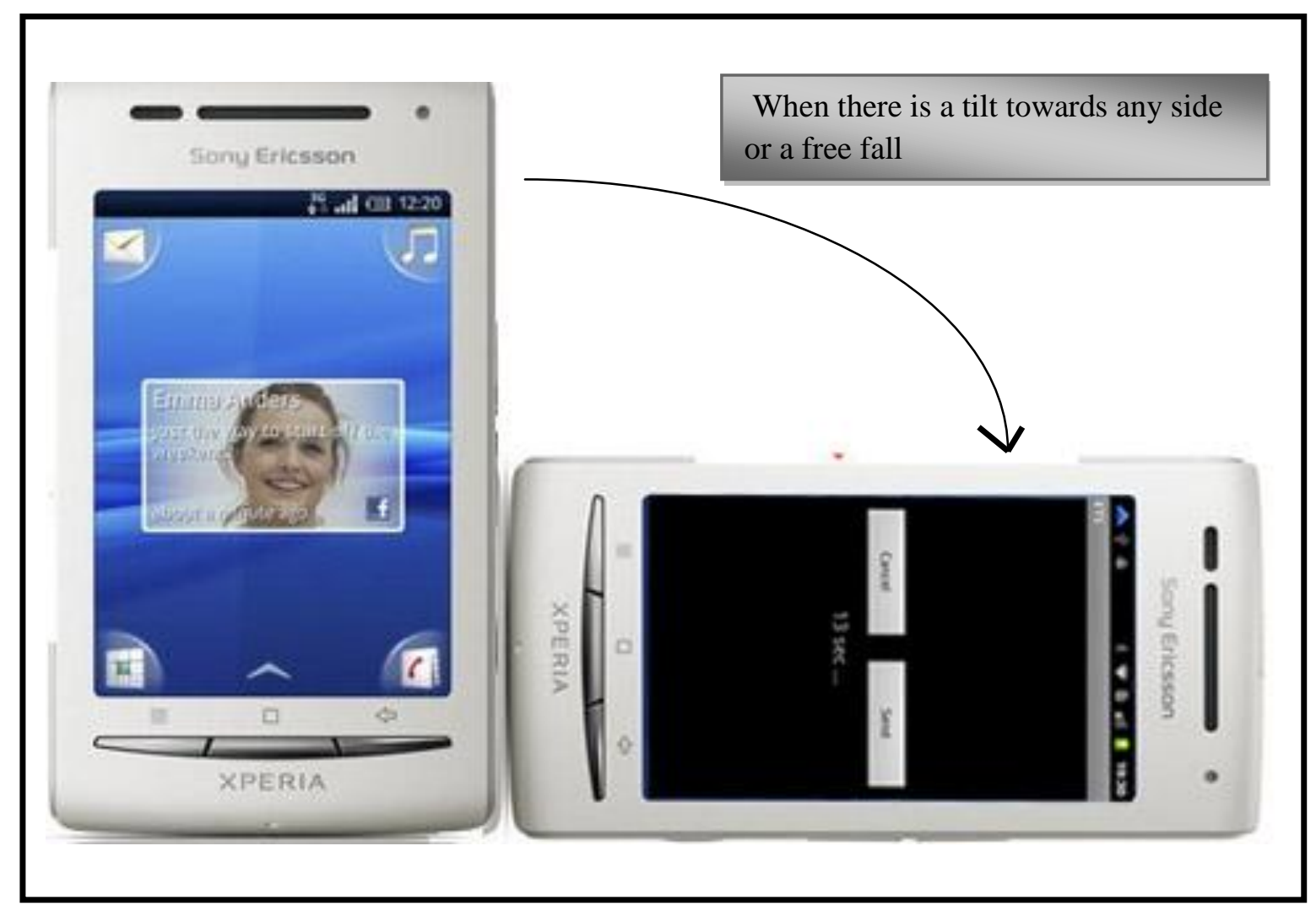

Figure 22 Sensing the movement of mobile using Accelerometer 


\section{Result}

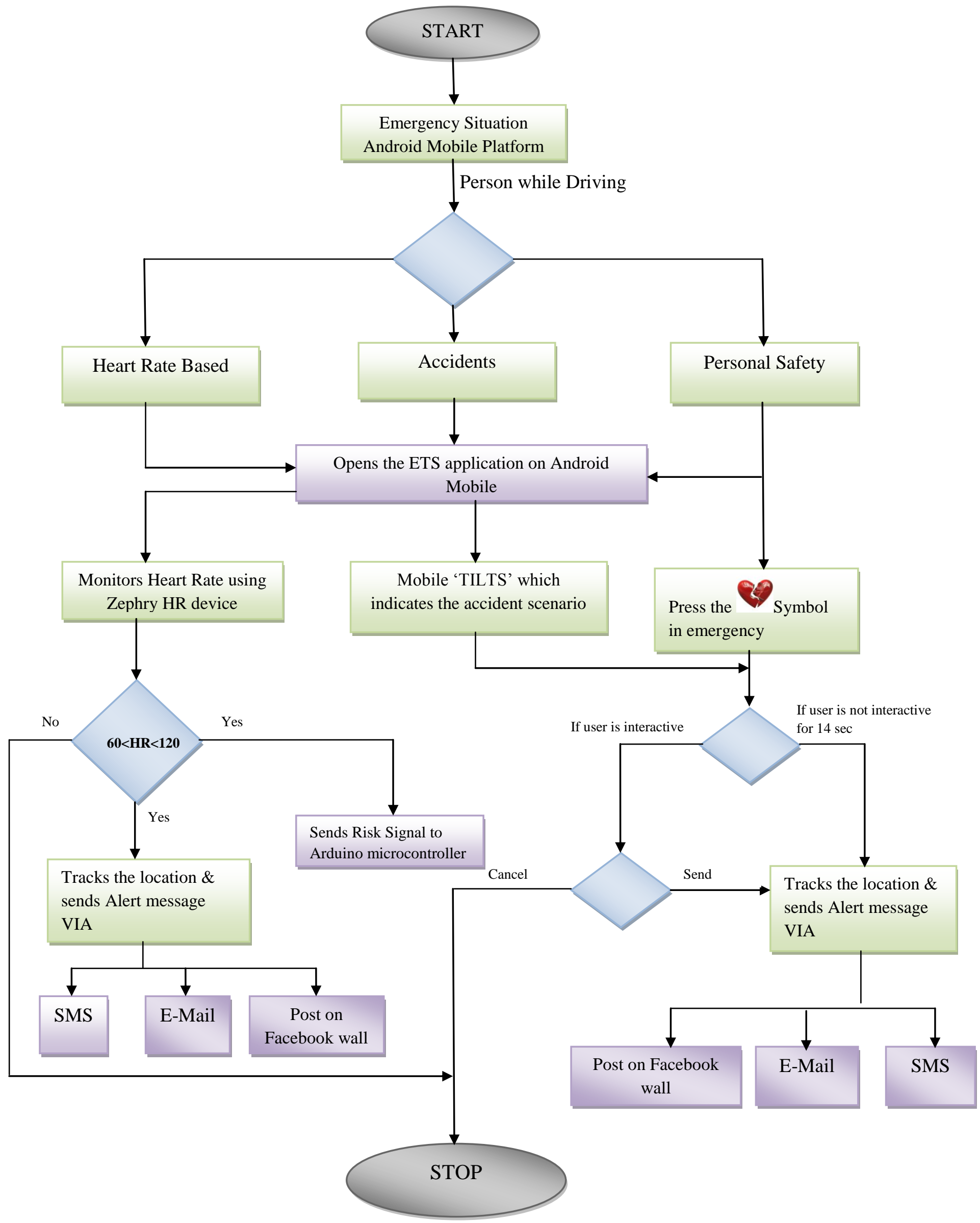

Figure 23 Working and Result of ETS Application 


\section{Conclusion}

Our research which was based on Emergency Tracking System using Google's Android Mobile platform and application based on that can achieve all the services and process as show in figure 23. Emergency situation was well sensed by the Android mobile with regards to the heart rate based, accidents and personal safety. Each individual emergency scenario was researched, designed and developed. This thesis was entirely targeted towards an emergency situation for a person who is driving a vehicle. Person's heart beat was monitored using a specialized HR device which sends the heart beat rate to the mobile which in turn makes a decision with regards to the abnormal heart rate and sends an alert signal to the arduino microcontroller, simultaneously a message was sent to a prestored number, email address and was posted on Facebook wall successfully.

Similarly accident based emergency scenario was also sensed by our intelligent application in Android mobile using a accelerometer and alert message which contains the GPS location information was sent via SMS, email and message was successfully posted on respective user's Facebook wall. Personal safety was also a major issue which we dealt and researched. In this thesis we also answered to this by integrating a user interactive one touch button which will track the location information and send those as a message via SMS, email and same will be posted on user's Facebook wall. Hence, Android once again proved to be a versatile operating system which allowed us to manipulate various inbuilt features of an Android mobile which made us to develop an intelligent application called as ETS. 


\section{REFERENCES}

[1] Gozalvez, Javier "First Google's android phone launched", IEEE Vehicular Technology Magazine, vol. 3, Sept 2008.

[2] [Online] Available: http://www.xcubelabs.com/evolution-of-mobile-operating-systems.php [accessed on June 10, 2011.]

[3] J.P Conti, "The Androids are coming," IEEE Engineering and Technology, vol.3 (9), pp.72-7, Sept. 2008.

[4] Open Handset Alliance, Open Handset Announces 14 New Members, [Online]. Available: http://www.openhandsetalliance.com/android_overview.html [accessed on June 10, 2011.]

[5] Xinhua Shu, Zhenjun Du and Rong Chen, "Research on Mobile Location Service Design Based on Android,"5th Int. Conf. On Wireless Communication Networking and Mobile Computing, Beijing, 2009, pp. 14.

[6] J. Whipple, W. Arensman and M.S Boler, "A Public Safety Application of GPS-Enabled smart phones and the Android Operating System," IEEE Int. Conf. on System, Man and Cybernetics, San Antonio, 2009, pp. 2059-2061.

[7] Chao Tung Yang, Yen Yu Chu and Shyh Chang Tsaur, "Implementation of a medical information service on Android mobile device," in $4^{\text {th }}$ Int. Conf. On News Trends in Information Science and Service Science. Gyeongju, 2010, pp. 72-77.

[8] Qun Hou, "Research and implementation of remote heart rate monitoring system based on GSM and MCU," in $2^{\text {nd }}$ Int. Conf. On Information Science and Engineering, Hangzhou, 2010, pp.2293.

[9] [Online] Available: Http://developer.android.com/reference [accessed on June 10, 2011.]

[10] Jiangpeng Dai, Jin Teng, Xiaole Bai, Zhaohui Shen and Dong Xuan, "Mobile phone based drunk driving detection," in 4th Int. Conf. On Pervasive Computing Technologies for Healthcare 2010, pp.1-8, 22-25.

[11] Yong Hua Cheng, Wen Kuang Kuo and Szu Lin Su, "An Android system design and implementation for Telematics services, " in $5^{\text {th }}$ Int. Conf. on Intelligent Computing and Intelligent Systems. 2010. Xiamen.pp.206

[12] Sheng-Yuan Yang, Dong-Liang Lee and Kune-Yao Chen, "A new ubiquitous information agent system for cloud computing - Example on GPS and Bluetooth techniques in Google Android platform," in Int. Conf. On Electric Information and Control Engineering, 2011, pp.1929-1932, 15-17.

[13] [Online] Available: Http://developer.android.com/guide [accessed on June 10, 2011.]

[14] Zhijun Wu, Jiaming He and Xingbin Zeng, "Key technology research on adaptation layer of android platform," in 2011 Int. Conf. On Consumer Electronics, Communications and Networks ,pp.4154-4156, 16-18, April2011.

[15] Y Kato, "Splish: A Visual Programming Environment for Arduino to Accelerate Physical Computing Experiences," in 8th Int. Conf. on Creating Connecting and Collaborating through Computing, pp.3-10, 25-28, January 2010.

[16] D. A. Mellis, M. Banzi, D. Cuartielles, and T. Igoe, "Arduino: An Open Electronics Prototyping Platform," ACM Conf. on Human Factors in Computing Systems, Apr. 2007.

[17] [Online] Available: Http://www.arduino.cc [accessed on June 10, 2011.]

[18] [Online] Available: Http://www.amarino-toolkit.net [accessed on June 10, 2011.] 
[19] M.R Neuman , "Vital Signs: Heart Rate," Pulse, IEEE, vol.1, no.3, pp.51-55, Dec.2010

[20] Yaofeng Wen, Rong Yang and Yuquan Chen, "Heart rate monitoring in dynamic movements from a wearable system, " in 5th Intl. Summer School and Symposium on Medical Devices and Biosensors, , pp. 272-275, 1-3, June 2008.

[21] N.H Mahmood, N. Uyop, N. Zulkarnain, F.K.C Harun, M.F Kamarudin and A. Linoby, "LED indicator for heart rate monitoring system in sport application," IEEE 7th Int. Colloquium on Signal Processing and its Applications, pp.64-66, 4-6. 2011.

[22] [Online] Available: Http:// www.heartratemonitor-app.co.uk/index.html [accessed on June 10,2011.] 